\title{
On a fractional hybrid multi-term integro-differential inclusion with four-point sum and integral boundary conditions
}

\author{
Dumitru Baleanu ${ }^{1,4}$, Sina Etemad ${ }^{2}$ and Shahram Rezapour ${ }^{2,3^{*}}$ (D)
}

\author{
"Correspondence: \\ rezapourshahram@yahoo.ca; \\ sh.rezapour@azaruniv.ac.ir \\ ${ }^{2}$ Department of Mathematics, \\ Azarbaijan Shahid Madani \\ University, Tabriz, Iran \\ ${ }^{3}$ Department of Medical Research, \\ China Medical University Hospital, \\ China Medical University, Taichung, \\ Taiwan \\ Full list of author information is \\ available at the end of the article
}

\begin{abstract}
We investigate the existence of solutions for a fractional hybrid multi-term integro-differential inclusion with four-point sum and integral boundary value conditions. By using Dhage's fixed point results, we prove our main existence result. Finally, we give an example to illustrate our main result.

MSC: Primary 34A08; secondary 34A12

Keywords: Four-point sum boundary value condition; Fractional hybrid inclusion problem; Dhage's fixed point result; Fractional Caputo derivative
\end{abstract}

\section{Introduction}

One possible way that the mathematics can help the various fields of science is to become more powerful and flexible in modeling theory so that different types of phenomena with distinct parameters can be written in mathematical formulas. In this case, different types of software can be developed to allow for more cost-free testing and less material consumption. One of the basic methods in this way is working with fractional calculus and investigating different mathematical modelings based on fractional operators in fractional boundary value problems with applied boundary conditions. Nowadays, many researchers are studying different types of integro-differential equations [1-7] or inclusions [8, 9], qdifferences [10-13], approximate solutions [14-20], the hybrid equations [21-28], and advanced fractional modelings [29-34].

The starting point for this field was a work of Dhage and Lakshmikantham in 2010 [35]. They introduced a new category of nonlinear differential equation called ordinary hybrid differential equation and studied the existence of extremal solutions for this boundary value problem by establishing some fundamental differential inequalities [35]. In 2012, Zhao et al. provided an extension for Dhage's work to fractional order and considered a boundary value problem of fractional hybrid differential equations [36]. Later, some papers on different properties of solutions for fractional hybrid boundary value problems were published. In 2015, Hilal and Kajouni discussed the existence of extremal solutions

(c) The Author(s) 2020. This article is licensed under a Creative Commons Attribution 4.0 International License, which permits use, sharing, adaptation, distribution and reproduction in any medium or format, as long as you give appropriate credit to the original author(s) and the source, provide a link to the Creative Commons licence, and indicate if changes were made. The images or other third party material in this article are included in the article's Creative Commons licence, unless indicated otherwise in a credit line to the material. If material is not included in the article's Creative Commons licence and your intended use is not permitted by statutory regulation or exceeds the permitted use, you will need to obtain permission directly from the copyright holder. To view a copy of this licence, visit http://creativecommons.org/licenses/by/4.0/. 
for the Caputo hybrid boundary value problem

$$
\left\{\begin{array}{l}
c \mathcal{D}_{0}^{p}\left(\frac{k(t)}{h(t, k(t))}\right)=g(t, k(t))=0, \\
a \frac{k(0)}{h(0, k(0))}+b \frac{k(T)}{h(T, k(T))}=c
\end{array}\right.
$$

where $t \in J=[0, T], p \in(0,1)$, the functions $h: J \times \mathbb{R} \rightarrow \mathbb{R} \backslash\{0\}$ and $g: J \times \mathbb{R} \rightarrow \mathbb{R}$ are continuous, and $a, b, c \in \mathbb{R}$ with $a+b \neq 0$ [37]. In 2016, Ahmad et al. studied the existence of solutions for the nonlocal boundary value problem of fractional hybrid inclusion problem

$$
\left\{\begin{array}{l}
{ }^{c} \mathcal{D}_{0}^{\alpha}\left(\frac{k(t)-\sum_{i=1}^{m} \mathcal{I}_{0}^{\beta_{i}} h_{i}(t, k(t))}{g(t, k(t))}\right) \in \mathcal{G}(t, k(t))=0, \\
k(0)=\mu(x), \quad k(1)=A \in \mathbb{R},
\end{array}\right.
$$

where $t \in J=[0,1],{ }^{c} \mathcal{D}_{0}^{\alpha}$ denotes the Caputo fractional derivative of order $\alpha \in(1,2]$, and $\mathcal{I}_{0}^{\phi}$ is the Riemann-Liouville fractional integral of order $\phi>0$ with $\phi \in\left\{\beta_{1}, \beta_{2}, \ldots, \beta_{m}\right\}$ [38]. In the same year, Baleanu et al. investigated some existence results and the dimension of the solution set for the fractional hybrid inclusion problem

$$
{ }^{c} \mathcal{D}^{v}\left(\frac{k(t)}{\Lambda\left(t, k(t), \mathcal{I}^{\alpha_{1}} k(t), \ldots, \mathcal{I}^{\alpha_{n}} k(t)\right)}\right) \in \Psi\left(t, k(t), \mathcal{I}^{\beta_{1}} k(t), \ldots, \mathcal{I}^{\beta_{m}} k(t)\right),
$$

with boundary value conditions $k(0)=k_{0}^{*}$ and $k(1)=k_{1}^{*}$, where $t \in[0,1], v \in(1,2],{ }^{c} \mathcal{D}^{v}$ and $\mathcal{I}^{\gamma}$ denote the Caputo derivative operator of the fractional order $v$ and the RiemannLiouville integral operator of the fractional order $\gamma \in\left\{\alpha_{i}, \beta_{j}\right\} \subset(0, \infty)$ for $i=1, \ldots n$ and $j=1, \ldots, m$, respectively [8]. In 2019, Derbazi et al. studied the existence and uniqueness results for the fractional hybrid boundary value problem

$$
\left\{\begin{array}{l}
{ }^{c} \mathcal{D}^{\alpha}\left(\frac{k(t)-h(t, k(t))}{w(t, k(t))}\right)=\Theta(t, k(t)), \\
\left.a_{1}\left(\frac{k(t)-h(t, k(t))}{w(t, k(t))}\right)\right|_{t=0}+\left.b_{1}\left(\frac{k(t)-h(t, k(t))}{w(t, k(t))}\right)\right|_{t=T}=\lambda_{1}, \\
\left.a_{2}{ }^{c} \mathcal{D}^{\beta}\left(\frac{k(t)-h(t, k(t))}{w(t, k(t))}\right)\right|_{t=\eta}+\left.b_{2}{ }^{c} \mathcal{D}^{\beta}\left(\frac{k(t)-h(t, k(t))}{w(t, k(t))}\right)\right|_{t=T}=\lambda_{2},
\end{array}\right.
$$

where $t \in[0, T], \alpha \in(1,2], \beta \in(0,1], \eta \in(0, T), a_{1}, a_{2}, b_{1}, b_{2}, \lambda_{1}, \lambda_{2} \in \mathbb{R}$, and the fractional derivatives that appeared are Caputo-type ones [39].

By using the idea of these works, we investigate the fractional hybrid multi-term integrodifferential inclusion of Caputo type

$$
{ }^{C} \mathcal{D}_{0}^{\omega}\left(\frac{k(t)}{\xi\left(t, k(t), \int_{0}^{1} k(s) \mathrm{d} s\right)}\right) \in \mathcal{S}\left(t, k(t), \phi_{1}(k(t)), \ldots, \phi_{m}(k(t))\right),
$$

with four-point sum and integral hybrid boundary value conditions

$$
\left\{\begin{array}{l}
\left.\left(\frac{k(t)}{\xi\left(t, k(t), \int_{0}^{1} k(s) \mathrm{d} s\right)}\right)\right|_{t=0}+\left.\sum_{j=1}^{r} b_{j} \mathcal{D}_{0}\left(\frac{k(t)}{\xi\left(t, k(t), \int_{0}^{1} k(s) \mathrm{d} s\right)}\right)\right|_{t=\eta_{1}}=0, \\
\left.\lambda_{1}\left(\frac{k(t)}{\xi\left(t, k(t), \int_{0}^{1} k(s) \mathrm{d} s\right)}\right)\right|_{t=\eta_{2}}+\left.\lambda_{2} \sum_{j=1}^{r} b_{j} \mathcal{D}_{0}^{2}\left(\frac{k(t)}{\xi\left(t, k(t), \int_{0}^{1} k(s) \mathrm{d} s\right)}\right)\right|_{t=1}=0 \\
\lambda_{3} \int_{0}^{1}\left(\frac{k(t)}{\xi\left(t, k(t), \int_{0}^{1} k(s) \mathrm{d} s\right)}\right) \mathrm{d} s=0
\end{array}\right.
$$

where $t \in[0,1], 0<\eta_{1}<\eta_{2}<1,{ }^{C} \mathcal{D}_{0}^{\omega}$ denotes the Caputo fractional derivative of order $\omega \in(2,3], \xi \in C([0,1] \times \mathbb{R} \times \mathbb{R}, \mathbb{R} \backslash\{0\}), \mathcal{S}:[0,1] \times \mathbb{R}^{m+1} \rightarrow \mathcal{P}(\mathbb{R})$ is a set-valued map 
via some properties, and $\lambda_{1}, \lambda_{2}, \lambda_{3}, b_{j} \in \mathbb{R}^{+}$for $j=1, \ldots, r$. Moreover, for each $i=1, \ldots, m$, assume that $\phi_{i}(k(t))=\int_{0}^{t} \frac{(t-s)^{\omega_{i}-1}}{\Gamma\left(\varpi_{i}\right)} k(s) \mathrm{d} s$ with $\varpi_{i}>0$.

\section{Preliminaries}

Let $\omega>0$. The Riemann-Liouville fractional integral of a function $k:[a, b] \rightarrow \mathbb{R}$ is defined by $\mathcal{I}_{0}^{\omega} k(t)=\int_{0}^{t} \frac{(t-s)^{\omega-1}}{\Gamma(\omega)} k(s) \mathrm{d} s$ provided that the right-hand side integral exists $([40,41])$. Now, let $n-1<\omega<n$ and $n=[\omega]+1$. The Caputo fractional derivative of a function $k \in$ $C^{(n)}([a, b], \mathbb{R})$ is defined by ${ }^{C} \mathcal{D}_{0}^{\omega} k(t)=\int_{0}^{t} \frac{(t-s)^{n-\omega-1}}{\Gamma(n-\omega)} k^{(n)}(s) \mathrm{d} s$ provided that the right-hand side integral exists $([40,41])$. It has been proved that the general solution for the homogeneous fractional differential equation ${ }^{C} \mathcal{D}_{0}^{\omega} k(t)=0$ is in the form $k(t)=m_{0}^{*}+m_{1}^{*} t+m_{2}^{*} t^{2}+\cdots+$ $m_{n-1}^{*} t^{n-1}$, and we have

$$
\mathcal{I}_{0}^{\omega}\left({ }^{C} \mathcal{D}_{0}^{\omega} k(t)\right)=k(t)+\sum_{j=0}^{n-1} m_{j}^{*} t^{j}=k(t)+m_{0}^{*}+m_{1}^{*} t+m_{2}^{*} t^{2}+\cdots+m_{n-1}^{*} t^{n-1}
$$

where $m_{0}^{*}, \ldots, m_{n-1}^{*}$ are some real constants and $n=[\omega]+1$ [42].

Assume that $(\mathcal{X},\|\cdot\| \mathcal{X})$ is a normed space. The set of all subsets of $\mathcal{X}$, the set of all closed subsets of $\mathcal{X}$, the set of all bounded subsets of $\mathcal{X}$, the set of all compact subsets of $\mathcal{X}$, and the set of all convex subsets of $\mathcal{X}$ are represented by $\mathcal{P}(\mathcal{X}), \mathcal{P}_{\mathrm{cl}}(\mathcal{X}), \mathcal{P}_{b}(\mathcal{X}), \mathcal{P}_{\mathrm{cp}}(\mathcal{X})$, and $\mathcal{P}_{\text {cv }}(\mathcal{X})$, respectively. We say that $k^{*} \in \mathcal{X}$ is a fixed point for the set-valued map $\mathcal{S}: \mathcal{X} \rightarrow$ $\mathcal{P}(\mathcal{X})$ if $k^{*} \in \mathcal{S}\left(k^{*}\right)$ [43]. The set of all fixed points of the set-valued map $\mathcal{S}$ is denoted by $\mathcal{F} \mathcal{I} \mathcal{X}(\mathcal{S})$ [43]. The Pompeiu-Hausdorff metric $\mathrm{PH}_{d}: \mathcal{P}(\mathcal{X}) \times \mathcal{P}(\mathcal{X}) \rightarrow \mathbb{R} \cup\{\infty\}$ is defined by

$$
\mathrm{PH}_{d_{\mathcal{X}}}\left(A_{1}, A_{2}\right)=\max \left\{\sup _{a_{1} \in A_{1}} d_{\mathcal{X}}\left(a_{1}, A_{2}\right), \sup _{a_{2} \in A_{2}} d_{\mathcal{X}}\left(A_{1}, a_{2}\right)\right\}
$$

where $d_{\mathcal{X}}\left(A_{1}, a_{2}\right)=\inf _{a_{1} \in A_{1}} d_{\mathcal{X}}\left(a_{1}, a_{2}\right)$ and $d_{\mathcal{X}}\left(a_{1}, A_{2}\right)=\inf _{a_{2} \in A_{2}} d_{\mathcal{X}}\left(a_{1}, a_{2}\right)$ [43]. A setvalued map $\mathcal{S}: \mathcal{X} \rightarrow \mathcal{P}_{\mathrm{cl}}(\mathcal{X})$ is said to be Lipschitz with constant $\lambda^{*}>0$ whenever we have $\mathrm{PH}_{d_{\mathcal{X}}}\left(\mathcal{S}\left(k_{1}\right), \mathcal{S}\left(k_{2}\right)\right) \leq \lambda^{*} d_{\mathcal{X}}\left(k_{1}, k_{2}\right)$ for all $k_{1}, k_{2} \in \mathcal{X}$. A Lipschitz map $\mathcal{S}$ is called contraction whenever $\lambda^{*} \in(0,1)$ [43]. We say that the set-valued map $\mathcal{S}$ is completely continuous whenever the set $\mathcal{S}(W)$ is relatively compact for every $W \in \mathcal{P}_{b}(\mathcal{X})$. A setvalued map $\mathcal{S}:[0,1] \rightarrow \mathcal{P}_{\mathrm{cl}}(\mathbb{R})$ is said to be measurable if the function $t \longmapsto d_{\mathcal{X}}(v, \mathcal{S}(t))$ is measurable for all $v \in \mathbb{R}[43,44]$. We say that the set-valued map $\mathcal{S}$ is upper semicontinuous (u.s.c.) whenever, for each $k^{*} \in \mathcal{X}$, the set $\mathcal{S}\left(k^{*}\right)$ belongs to $\mathcal{P}_{\mathrm{cl}}(\mathcal{X})$, and for every open set $\mathcal{V}$ containing $\mathcal{S}\left(k^{*}\right)$, there exists an open neighborhood $\mathcal{U}_{0}^{*}$ of $k^{*}$ such that $\mathcal{S}\left(\mathcal{U}_{0}^{*}\right) \subseteq \mathcal{V}$ [43]. The graph of the set-valued map $\mathcal{S}: \mathcal{X} \rightarrow \mathcal{P}_{\mathrm{cl}}(\mathcal{Y})$ is defined by $\operatorname{Graph}(\mathcal{S})=\{(k, s) \in \mathcal{X} \times \mathcal{Y}: s \in \mathcal{S}(k)\}$. We say that graph of $\mathcal{S}$ is a closed set if, for each sequence $\left\{k_{n}\right\}_{n \geq 1}$ in $\mathcal{X}$ and $\left\{s_{n}\right\}_{n \geq 1}$ in $\mathcal{Y}, k_{n} \rightarrow k_{0}, s_{n} \rightarrow s_{0}$ and $s_{n} \in \mathcal{S}\left(k_{n}\right)$, we have $s_{0} \in \mathcal{S}\left(k_{0}\right)$ $[43,44]$. Suppose that the set-valued map $\mathcal{S}: \mathcal{X} \rightarrow \mathcal{P}_{\mathrm{cl}}(\mathcal{Y})$ is upper semi-continuous. Then $\operatorname{Graph}(\mathcal{S})$ is a subset of the product space $\mathcal{X} \times \mathcal{Y}$ which is a closed set. Conversely, if the set-valued map $\mathcal{S}$ is completely continuous and has a closed graph, then $\mathcal{S}$ is upper semicontinuous ([43], Proposition 2.1). A set-valued map $\mathcal{S}$ is convex-valued if $\mathcal{S}(k)$ is a convex set for each element $k \in \mathcal{X}$. A set of selections of set-valued map $\mathcal{S}$ at point $k \in C([0,1], \mathbb{R})$ is defined by

$$
(\mathcal{S E} \mathcal{L})_{\mathcal{S}, k}:=\left\{\vartheta \in \mathcal{L}^{1}([0,1], \mathbb{R}): \vartheta(t) \in \mathcal{S}(t, k(t))\right\}
$$


for almost all $t \in[0,1][43,44]$. If $\mathcal{S}$ is an arbitrary set-valued map, then for each function $k \in C([0,1], \mathcal{X})$, we have $(\mathcal{S E L})_{\mathcal{S}, k} \neq \emptyset$ whenever $\operatorname{dim} \mathcal{X}<\infty$ [43]. A set-valued map $\mathcal{S}$ : $[0,1] \times \mathbb{R} \rightarrow \mathcal{P}(\mathbb{R})$ is called Caratheodory whenever $t \mapsto \mathcal{S}(t, k)$ is a measurable mapping for each function $k \in \mathbb{R}$ and $k \mapsto \mathcal{S}(t, k)$ is an upper semi-continuous mapping for almost all $t \in[0,1][43,44]$. Moreover, a Caratheodory set-valued map $\mathcal{S}:[0,1] \times \mathbb{R} \rightarrow \mathcal{P}(\mathbb{R})$ is said to be $\mathcal{L}^{1}$-Caratheodory whenever, for each constant $\mu>0$, there exists a function $\phi_{\mu} \in \mathcal{L}^{1}\left([0,1], \mathbb{R}^{+}\right)$such that $\|\mathcal{S}(t, k)\|=\sup _{t \in[0,1]}\{|q|: q \in \mathcal{S}(t, k)\} \leq \phi_{\mu}(t)$ for all $|k| \leq \mu$ and for almost all $t \in[0,1][43,44]$. We need the next results.

Theorem 1 ([45]) Suppose that $\mathcal{X}$ is a separable Banach space, $\mathcal{S}:[0,1] \times \mathcal{X} \rightarrow \mathcal{P}_{\mathrm{cp}, \mathrm{cv}}(\mathcal{X})$ is an $\mathcal{L}^{1}$-Carathéodory set-valued map, and $\Xi: \mathcal{L}^{1}([0,1], \mathcal{X}) \rightarrow C([0,1], \mathcal{X})$ is a linear continuous mapping. Then the composition $\Xi \circ(\mathcal{S E} \mathcal{L})_{\mathcal{S}}: C([0,1], \mathcal{X}) \rightarrow \mathcal{P}_{\mathrm{cp}, \mathrm{cv}}(C([0,1], \mathcal{X}))$ is an operator in the product space $C([0,1], \mathcal{X}) \times C([0,1], \mathcal{X})$ with action $k \mapsto(\Xi \circ$ $\left.(\mathcal{S E} \mathcal{L})_{\mathcal{S}}\right)(k)=\Xi\left((\mathcal{S E L})_{\mathcal{S}, k}\right)$ having the closed graph property.

Theorem 2 ([46]) Let $\mathcal{X}$ be a Banach algebra. Assume that there exist a single-valued map $\Phi_{1}: \mathcal{X} \rightarrow \mathcal{X}$ and a set-valued map $\Phi_{2}: \mathcal{X} \rightarrow \mathcal{P}_{\text {cp,cv }}(\mathcal{X})$ such that

(i) $\Phi_{1}$ is an operator including the Lipschitzian property with a Lipschitz constant $l^{*}$;

(ii) $\Phi_{2}$ is an operator including upper semi-continuity and the compactness property;

(iii) $2 l^{*} \hat{\Delta}<1$ such that $\hat{\Delta}=\left\|\Phi_{2}(\mathcal{X})\right\|$.

Then either the set $\mathcal{O}^{*}=\left\{v^{*} \in \mathcal{X} \mid \alpha_{0} v^{*} \in \Phi_{1} v^{*} \Phi_{2} v^{*}, \alpha_{0}>1\right\}$ is unbounded or there is a solution in $\mathcal{X}$ for the operator inclusion $k \in \Phi_{1} k \Phi_{2} k$.

\section{Main results}

Now, we are ready to study the fractional hybrid multi-term inclusion problem (1)(2). Consider the Banach space $\mathcal{X}=\left\{k(t): k(t) \in C_{\mathbb{R}}([0,1])\right\}$ with the norm $\|k\|_{\mathcal{X}}=$ $\sup _{t \in[0,1]}|k(t)|$. For convenience, consider the constants

$$
\begin{aligned}
& \Lambda_{0}=\frac{2 \lambda_{1} \eta_{1} \sum_{j=1}^{r} b_{j}-\lambda_{1} \eta_{2}^{2}-2 \lambda_{2} \sum_{j=1}^{r} b_{j}}{\lambda_{1}}, \quad \Lambda_{5}=\frac{1}{\sum_{j=1}^{r} b_{j}-\eta_{2}}, \\
& \Lambda_{1}=\left(6 \eta_{2}\left(\lambda_{1} \eta_{1}+\lambda_{2}\right)+\lambda_{1}\left(2-3 \eta_{1}\right)\right) \sum_{j=1}^{r} b_{j}, \quad \Lambda_{6}=\frac{\lambda_{1} \eta_{2}^{2}+2 \lambda_{2} \sum_{j=1}^{r} b_{j}}{\lambda_{1}}, \\
& \Lambda_{2}=\left(3 \lambda_{1} \eta_{2}^{2}+6 \lambda_{2} \sum_{j=1}^{r} b_{j}\right)\left(1-2 \sum_{j=1}^{r} b_{j}\right)-2 \lambda_{1} \eta_{2}, \quad \Lambda_{7}=\lambda_{1}\left(\Lambda_{6}-\eta_{2} \Lambda_{0} \Lambda_{5}\right), \\
& \Lambda_{3}=\frac{3\left(2 \eta_{2}-1\right)}{\Lambda_{1}+\Lambda_{2}}, \quad \Lambda_{8}=\left[\lambda_{1}\left(\Lambda_{3}+\Lambda_{4}\right)\left[\eta_{2} \Lambda_{0} \Lambda_{5}-\Lambda_{6}\right]-\left(\eta_{2} \Lambda_{5}+1\right)\right] . \\
& \Lambda_{4}=\frac{6\left(\sum_{j=1}^{r} b_{j}-\eta_{2}\right)}{\Lambda_{1}+\Lambda_{2}} .
\end{aligned}
$$

Here, we prove our first key result.

Lemma 3 Let $z \in \mathcal{X}$. Then $k_{0}$ is a solution for the fractional hybrid differential equation

$$
{ }^{C} \mathcal{D}_{0}^{\omega}\left(\frac{k(t)}{\xi\left(t, k(t), \int_{0}^{1} k(s) \mathrm{d} s\right)}\right)=z(t), \quad(t \in[0,1], \omega \in(2,3])
$$


with four-point hybrid integral boundary value conditions

$$
\left\{\begin{array}{l}
\left.\left(\frac{k(t)}{\xi\left(t, k(t), \int_{0}^{1} k(s) \mathrm{d} s\right)}\right)\right|_{t=0}+\left.\sum_{j=1}^{r} b_{j} \mathcal{D}_{0}\left(\frac{k(t)}{\xi\left(t, k(t), \int_{0}^{1} k(s) \mathrm{d} s\right)}\right)\right|_{t=\eta_{1}}=0, \\
\left.\lambda_{1}\left(\frac{k(t)}{\xi\left(t, k(t), \int_{0}^{1} k(s) \mathrm{d} s\right)}\right)\right|_{t=\eta_{2}}+\left.\lambda_{2} \sum_{j=1}^{r} b_{j} \mathcal{D}_{0}^{2}\left(\frac{k(t)}{\xi\left(t, k(t), \int_{0}^{1} k(s) \mathrm{d} s\right)}\right)\right|_{t=1}=0, \\
\lambda_{3} \int_{0}^{1}\left(\frac{k(t)}{\xi\left(t, k(t), \int_{0}^{1} k(s) \mathrm{d} s\right)}\right) \mathrm{d} s=0
\end{array}\right.
$$

if and only if $k_{0}$ is a solution for the integral equation

$$
\begin{aligned}
k(t)= & \xi\left(t, k(t), \int_{0}^{1} k(s) \mathrm{d} s\right)\left[\frac{1}{\Gamma(\omega)} \int_{0}^{t}(t-s)^{\omega-1} z(s) \mathrm{d} s\right. \\
& +\frac{\left[\left(\Lambda_{3} \Lambda_{7}-\eta_{2} \Lambda_{5}\right)+t\left(\lambda_{1} \Lambda_{0} \Lambda_{3}-1\right) \Lambda_{5}-t^{2} \lambda_{1} \Lambda_{3}\right] \sum_{j=1}^{r} b_{j}}{\Gamma(\omega-1)} \\
& \times \int_{0}^{\eta_{1}}\left(\eta_{1}-s\right)^{\omega-2} z(s) \mathrm{d} s \\
& +\frac{1+\left(t \Lambda_{8}+\eta_{2}\right) \Lambda_{5}+\left(\Lambda_{3}+\Lambda_{4}\right)\left(t^{2} \lambda_{1}-\Lambda_{7}\right)}{\Gamma(\omega)} \int_{0}^{\eta_{2}}\left(\eta_{2}-s\right)^{\omega-1} z(s) \mathrm{d} s \\
& +\frac{\lambda_{2}\left[\left(1+t \Lambda_{5}\right) \Lambda_{8}+t^{2} \lambda_{1}\left(\Lambda_{3}+\Lambda_{4}\right)\right] \sum_{j=1}^{r} b_{j}}{\lambda_{1} \Gamma(\omega-2)} \int_{0}^{1}(1-s)^{\omega-3} z(s) \mathrm{d} s \\
& \left.+\frac{\lambda_{1} \Lambda_{4}\left[\left(t-\eta_{2}\right) \Lambda_{0} \Lambda_{5}-t^{2}-\Lambda_{6}\right]}{\Gamma(\omega)} \int_{0}^{1} \int_{0}^{s}(s-\tau)^{\omega-1} z(\tau) \mathrm{d} \tau \mathrm{d} s\right],
\end{aligned}
$$

where $\Lambda_{0}, \ldots, \Lambda_{8}$ are given in (3).

Proof Assume that $k_{0}$ is a solution for hybrid equation (4). Then there exist constants $m_{0}^{*}, m_{1}^{*}, m_{2}^{*} \in \mathbb{R}$ such that $\frac{k_{0}(t)}{\xi\left(t, k_{0}(t), \int_{0}^{1} k_{0}(s) \mathrm{d} s\right)}=\mathcal{I}_{0}^{\omega} z(t)+m_{0}^{*}+m_{1}^{*} t+m_{2}^{*} t^{2}$. Hence,

$$
k_{0}(t)=\xi\left(t, k_{0}(t), \int_{0}^{1} k_{0}(s) \mathrm{d} s\right)\left[\int_{0}^{t} \frac{(t-s)^{\omega-1}}{\Gamma(\omega)} z(s) \mathrm{d} s+m_{0}^{*}+m_{1}^{*} t+m_{2}^{*} t^{2}\right],
$$

and so

$$
\begin{aligned}
& \mathcal{D}_{0}\left(\frac{k_{0}(t)}{\xi\left(t, k_{0}(t), \int_{0}^{1} k_{0}(s) \mathrm{d} s\right)}\right)=\int_{0}^{t} \frac{(t-s)^{\omega-2}}{\Gamma(\omega-1)} z(s) \mathrm{d} s+m_{1}^{*}+2 m_{2}^{*} t, \\
& \mathcal{D}_{0}^{2}\left(\frac{k_{0}(t)}{\xi\left(t, k_{0}(t), \int_{0}^{1} k_{0}(s) \mathrm{d} s\right)}\right)=\int_{0}^{t} \frac{(t-s)^{\omega-3}}{\Gamma(\omega-2)} z(s) \mathrm{d} s+2 m_{2}^{*}, \\
& \int_{0}^{1}\left(\frac{k_{0}(t)}{\xi\left(t, k_{0}(t), \int_{0}^{1} k_{0}(s) \mathrm{d} s\right)}\right) \mathrm{d} s \\
& \quad=\int_{0}^{1} \int_{0}^{s} \frac{(s-\tau)^{\omega-1}}{\Gamma(\omega)} z(\tau) \mathrm{d} \tau \mathrm{d} s+m_{0}^{*}+\frac{1}{2} m_{1}^{*}+\frac{1}{3} m_{2}^{*} .
\end{aligned}
$$


By using the four-point hybrid boundary value conditions, we obtain

$$
\begin{aligned}
m_{0}^{*}= & \frac{\left(\Lambda_{3} \Lambda_{7}-\eta_{2} \Lambda_{5}\right) \sum_{j=1}^{r} b_{j}}{\Gamma(\omega-1)} \int_{0}^{\eta_{1}}\left(\eta_{1}-s\right)^{\omega-2} z(s) \mathrm{d} s \\
& -\frac{\Lambda_{7}\left(\Lambda_{3}+\Lambda_{4}\right)+\eta_{2} \Lambda_{5}+1}{\Gamma(\omega)} \int_{0}^{\eta_{2}}\left(\eta_{2}-s\right)^{\omega-1} z(s) \mathrm{d} s \\
& +\frac{\lambda_{2} \Lambda_{8} \sum_{j=1}^{r} b_{j}}{\lambda_{1} \Gamma(\omega-2)} \int_{0}^{1}(1-s)^{\omega-3} z(s) \mathrm{d} s \\
& -\frac{\lambda_{1} \Lambda_{4}\left(\eta_{2} \Lambda_{0} \Lambda_{5}+\Lambda_{6}\right)}{\Gamma(\omega)} \int_{0}^{1} \int_{0}^{s}(s-\tau)^{\omega-1} z(\tau) \mathrm{d} \tau \mathrm{d} s, \\
m_{1}^{*}= & \frac{\left(\lambda_{1} \Lambda_{0} \Lambda_{3}-1\right) \Lambda_{5} \sum_{j=1}^{r} b_{j}}{\Gamma(\omega-1)} \int_{0}^{\eta_{1}}\left(\eta_{1}-s\right)^{\omega-2} z(s) \mathrm{d} s \\
& +\frac{\left[1-\lambda_{1} \Lambda_{0}\left(\Lambda_{3}+\Lambda_{4}\right)\right] \Lambda_{5}}{\Gamma(\omega)} \int_{0}^{\eta_{2}}\left(\eta_{2}-s\right)^{\omega-1} z(s) \mathrm{d} s \\
& +\frac{\lambda_{2}\left[1-\lambda_{1} \Lambda_{0}\left(\Lambda_{3}+\Lambda_{4}\right)\right] \Lambda_{5} \sum_{j=1}^{r} b_{j}}{\lambda_{1} \Gamma(\omega-2)} \int_{0}^{1}(1-s)^{\omega-3} z(s) \mathrm{d} s \\
& +\frac{\lambda_{1} \Lambda_{0} \Lambda_{4} \Lambda_{5}}{\Gamma(\omega)} \int_{0}^{1} \int_{0}^{s}(s-\tau)^{\omega-1} z(\tau) \mathrm{d} \tau \mathrm{d} s,
\end{aligned}
$$

and

$$
\begin{aligned}
m_{2}^{*}= & -\frac{\lambda_{1} \Lambda_{3} \sum_{j=1}^{r} b_{j}}{\Gamma(\omega-1)} \int_{0}^{\eta_{1}}\left(\eta_{1}-s\right)^{\omega-2} z(s) \mathrm{d} s \\
& +\frac{\lambda_{1}\left(\Lambda_{3}+\Lambda_{4}\right)}{\Gamma(\omega)} \int_{0}^{\eta_{2}}\left(\eta_{2}-s\right)^{\omega-1} z(s) \mathrm{d} s \\
& +\frac{\lambda_{2}\left(\Lambda_{3}+\Lambda_{4}\right) \sum_{j=1}^{r} b_{j}}{\Gamma(\omega-2)} \int_{0}^{1}(1-s)^{\omega-3} z(s) \mathrm{d} s \\
& -\frac{\lambda_{1} \Lambda_{4}}{\Gamma(\omega)} \int_{0}^{1} \int_{0}^{s}(s-\tau)^{\omega-1} z(\tau) \mathrm{d} \tau \mathrm{d} s .
\end{aligned}
$$

By substituting the values $m_{0}^{*}, m_{1}^{*}$, and $m_{2}^{*}$ in (7), we get

$$
\begin{aligned}
k_{0}(t)= & \xi\left(t, k_{0}(t), \int_{0}^{1} k_{0}(s) \mathrm{d} s\right)\left[\frac{1}{\Gamma(\omega)} \int_{0}^{t}(t-s)^{\omega-1} z(s) \mathrm{d} s\right. \\
& +\frac{\left[\left(\Lambda_{3} \Lambda_{7}-\eta_{2} \Lambda_{5}\right)+t\left(\lambda_{1} \Lambda_{0} \Lambda_{3}-1\right) \Lambda_{5}-t^{2} \lambda_{1} \Lambda_{3}\right] \sum_{j=1}^{r} b_{j}}{\Gamma(\omega-1)} \\
& \times \int_{0}^{\eta_{1}}\left(\eta_{1}-s\right)^{\omega-2} z(s) \mathrm{d} s \\
& +\frac{1+\left(t \Lambda_{8}+\eta_{2}\right) \Lambda_{5}+\left(\Lambda_{3}+\Lambda_{4}\right)\left(t^{2} \lambda_{1}-\Lambda_{7}\right)}{\Gamma(\omega)} \int_{0}^{\eta_{2}}\left(\eta_{2}-s\right)^{\omega-1} z(s) \mathrm{d} s \\
& +\frac{\lambda_{2}\left[\left(1+t \Lambda_{5}\right) \Lambda_{8}+t^{2} \lambda_{1}\left(\Lambda_{3}+\Lambda_{4}\right)\right] \sum_{j=1}^{r} b_{j}}{\lambda_{1} \Gamma(\omega-2)} \int_{0}^{1}(1-s)^{\omega-3} z(s) \mathrm{d} s \\
& \left.+\frac{\lambda_{1} \Lambda_{4}\left[\left(t-\eta_{2}\right) \Lambda_{0} \Lambda_{5}-t^{2}-\Lambda_{6}\right]}{\Gamma(\omega)} \int_{0}^{1} \int_{0}^{s}(s-\tau)^{\omega-1} z(\tau) \mathrm{d} \tau \mathrm{d} s\right] .
\end{aligned}
$$


This shows that the function $k_{0}$ is a solution for integral equation (6). Conversely, one can easily check that $k_{0}$ is a solution for problem (4)-(5) whenever $k_{0}$ is a solution function for integral equation (6).

Definition 4 An absolutely continuous function $k:[0,1] \rightarrow \mathbb{R}$ is called a solution for the fractional hybrid inclusion problem (1)-(2) whenever there exists an integrable function $\vartheta \in \mathcal{L}^{1}([0,1], \mathbb{R})$ with $\vartheta(t) \in \mathcal{S}\left(t, k(t), \phi_{1}(k(t)), \ldots, \phi_{m}(k(t))\right)$ for almost all $t \in[0,1]$ satisfying the four-point fractional hybrid sum and integral boundary value conditions

$$
\left\{\begin{array}{l}
\left.\left(\frac{k(t)}{\xi\left(t, k(t), \int_{0}^{1} k(s) \mathrm{d} s\right)}\right)\right|_{t=0}+\left.\sum_{j=1}^{r} b_{j} \mathcal{D}_{0}\left(\frac{k(t)}{\xi\left(t, k(t), \int_{0}^{1} k(s) \mathrm{d} s\right)}\right)\right|_{t=\eta_{1}}=0, \\
\left.\lambda_{1}\left(\frac{k(t)}{\xi\left(t, k(t), \int_{0}^{1} k(s) \mathrm{d} s\right)}\right)\right|_{t=\eta_{2}}+\left.\lambda_{2} \sum_{j=1}^{r} b_{j} \mathcal{D}_{0}^{2}\left(\frac{k(t)}{\xi\left(t, k(t), \int_{0}^{1} k(s) \mathrm{d} s\right)}\right)\right|_{t=1}=0, \\
\lambda_{3} \int_{0}^{1}\left(\frac{k(t)}{\xi\left(t, k(t), \int_{0}^{1} k(s) \mathrm{d} s\right)}\right) \mathrm{d} s=0
\end{array}\right.
$$

and

$$
\begin{aligned}
k(t)= & \xi\left(t, k(t), \int_{0}^{1} k(s) \mathrm{d} s\right)\left[\frac{1}{\Gamma(\omega)} \int_{0}^{t}(t-s)^{\omega-1} \vartheta(s) \mathrm{d} s\right. \\
& +\frac{\left[\left(\Lambda_{3} \Lambda_{7}-\eta_{2} \Lambda_{5}\right)+t\left(\lambda_{1} \Lambda_{0} \Lambda_{3}-1\right) \Lambda_{5}-t^{2} \lambda_{1} \Lambda_{3}\right] \sum_{j=1}^{r} b_{j}}{\Gamma(\omega-1)} \\
& \times \int_{0}^{\eta_{1}}\left(\eta_{1}-s\right)^{\omega-2} \vartheta(s) \mathrm{d} s \\
& +\frac{1+\left(t \Lambda_{8}+\eta_{2}\right) \Lambda_{5}+\left(\Lambda_{3}+\Lambda_{4}\right)\left(t^{2} \lambda_{1}-\Lambda_{7}\right)}{\Gamma(\omega)} \int_{0}^{\eta_{2}}\left(\eta_{2}-s\right)^{\omega-1} \vartheta(s) \mathrm{d} s \\
& +\frac{\lambda_{2}\left[\left(1+t \Lambda_{5}\right) \Lambda_{8}+t^{2} \lambda_{1}\left(\Lambda_{3}+\Lambda_{4}\right)\right] \sum_{j=1}^{r} b_{j}}{\lambda_{1} \Gamma(\omega-2)} \int_{0}^{1}(1-s)^{\omega-3} \vartheta(s) \mathrm{d} s \\
& \left.+\frac{\lambda_{1} \Lambda_{4}\left[\left(t-\eta_{2}\right) \Lambda_{0} \Lambda_{5}-t^{2}-\Lambda_{6}\right]}{\Gamma(\omega)} \int_{0}^{1} \int_{0}^{s}(s-\tau)^{\omega-1} \vartheta(\tau) \mathrm{d} \tau \mathrm{d} s\right]
\end{aligned}
$$

for all $t \in[0,1]$.

Now, we provide our main result.

Theorem 5 Suppose that $\xi:[0,1] \times \mathbb{R} \times \mathbb{R} \rightarrow \mathbb{R} \backslash\{0\}$ is a continuous function and $\mathcal{S}$ : $[0,1] \times \mathbb{R}^{m+1} \rightarrow \mathcal{P}_{\mathrm{cp}, \mathrm{cv}}(\mathbb{R})$ is a set-valued map. Assume that

$(\mathcal{C} 1)$ there exists a bounded mapping $\theta:[0,1] \rightarrow \mathbb{R}^{+}$such that

$$
\left|\xi\left(t, k_{1}(t), k_{2}(t)\right)-\xi\left(t, k_{1}^{\prime}(t), k_{2}^{\prime}(t)\right)\right| \leq \theta(t) \sum_{i=1}^{2}\left|k_{i}(t)-k_{i}^{\prime}(t)\right|
$$

for all $k_{1}, k_{2}, k_{1}^{\prime}, k_{2}^{\prime} \in \mathbb{R}$, and $t \in[0,1]$;

$(\mathcal{C} 2)$ the set-valued map $\mathcal{S}:[0,1] \times \mathbb{R}^{m+1} \rightarrow \mathcal{P}_{\mathrm{cp}, \mathrm{cv}}(\mathbb{R})$ has the $\mathcal{L}^{1}$-Caratheodory property;

$(\mathcal{C} 3)$ there exists a positive mapping $q(t) \in \mathcal{L}^{1}\left([0,1], \mathbb{R}^{+}\right)$such that

$$
\left\|\mathcal{S}\left(t, k_{1}, k_{2}, \ldots, k_{m+1}\right)\right\|=\sup \left\{|\vartheta|: \vartheta \in \mathcal{S}\left(t, k_{1}(t), k_{2}(t), \ldots, k_{m+1}(t)\right)\right\} \leq q(t)
$$

for all $k_{1}, \ldots, k_{m+1} \in \mathbb{R}$ and for almost all $t \in[0,1]$; 
$(\mathcal{C} 4)$ there exists a positive real number $\tilde{\rho} \in \mathbb{R}$ such that

$$
\tilde{\rho}>\frac{\xi^{*} M\|q\|_{\mathcal{L}^{1}}}{1-2 \theta^{*} M\|q\|_{\mathcal{L}^{1}}}
$$

where $\|q\|_{\mathcal{L}^{1}}=\int_{0}^{1}|q(s)| \mathrm{d} s, \xi^{*}=\sup _{t \in[0,1]}|\xi(t, 0,0)|, \theta^{*}=\sup _{t \in[0,1]}|\theta(t)|$ and

$$
\begin{aligned}
M= & \frac{1}{\Gamma(\omega+1)} \\
& +\frac{\left[\left|\Lambda_{3} \Lambda_{7}\right|+\left|\eta_{2} \Lambda_{5}\right|+\left(\left|\lambda_{1} \Lambda_{0} \Lambda_{3}\right|+1\right)\left|\Lambda_{5}\right|+\left|\lambda_{1} \Lambda_{3}\right|\right] \eta_{1}^{\omega-1} \sum_{j=1}^{r} b_{j}}{\Gamma(\omega)} \\
& +\frac{\left[1+\left|\left(\Lambda_{8}+\eta_{2}\right) \Lambda_{5}\right|+\left|\left(\Lambda_{3}+\Lambda_{4}\right)\right|\left(\lambda_{1}+\left|\Lambda_{7}\right|\right)\right] \eta_{2}^{\omega}}{\Gamma(\omega+1)} \\
& +\frac{\lambda_{2}\left[\left|\left(1+\Lambda_{5}\right) \Lambda_{8}\right|+\lambda_{1}\left|\Lambda_{3}+\Lambda_{4}\right|\right] \sum_{j=1}^{r} b_{j}}{\lambda_{1} \Gamma(\omega-1)} \\
& +\frac{\lambda_{1}\left|\Lambda_{4}\right|\left[\left(1+\eta_{2}\right)\left|\Lambda_{0} \Lambda_{5}\right|+\left|\Lambda_{6}\right|+1\right]}{\Gamma(\omega+2)} .
\end{aligned}
$$

Then the hybrid inclusion problem (1)-(2) has a solution whenever $4 \theta^{*} M\|q\|_{\mathcal{L}^{1}}<1$.

Proof For each $k \in \mathcal{X}$, define the set of selections of the operator $\mathcal{S}$ by

$$
(\mathcal{S E} \mathcal{L})_{\mathcal{S}, k}=\left\{\vartheta \in \mathcal{L}^{1}([0,1]): \vartheta(t) \in \mathcal{S}\left(t, k(t), \phi_{1}(k(t)), \ldots, \phi_{m}(k(t))\right)\right\}
$$

for almost all $t \in[0,1]$. Define $\mathcal{G}: \mathcal{X} \rightarrow \mathcal{P}(\mathcal{X})$ by

$$
\mathcal{G}(k)=\{g \in \mathcal{X}: g(t)=a(t) \text { for } t \in[0,1]\},
$$

where

$$
\begin{aligned}
a(t)= & \xi\left(t, k(t), \int_{0}^{1} k(s) \mathrm{d} s\right)\left[\frac{1}{\Gamma(\omega)} \int_{0}^{t}(t-s)^{\omega-1} \vartheta(s) \mathrm{d} s\right. \\
& +\frac{\left[\left(\Lambda_{3} \Lambda_{7}-\eta_{2} \Lambda_{5}\right)+t\left(\lambda_{1} \Lambda_{0} \Lambda_{3}-1\right) \Lambda_{5}-t^{2} \lambda_{1} \Lambda_{3}\right] \sum_{j=1}^{r} b_{j}}{\Gamma(\omega-1)} \int_{0}^{\eta_{1}}\left(\eta_{1}-s\right)^{\omega-2} \vartheta(s) \mathrm{d} s \\
& +\frac{1+\left(t \Lambda_{8}+\eta_{2}\right) \Lambda_{5}+\left(\Lambda_{3}+\Lambda_{4}\right)\left(t^{2} \lambda_{1}-\Lambda_{7}\right)}{\Gamma(\omega)} \int_{0}^{\eta_{2}}\left(\eta_{2}-s\right)^{\omega-1} \vartheta(s) \mathrm{d} s \\
& +\frac{\lambda_{2}\left[\left(1+t \Lambda_{5}\right) \Lambda_{8}+t^{2} \lambda_{1}\left(\Lambda_{3}+\Lambda_{4}\right)\right] \sum_{j=1}^{r} b_{j}}{\lambda_{1} \Gamma(\omega-2)} \int_{0}^{1}(1-s)^{\omega-3} \vartheta(s) \mathrm{d} s \\
& \left.+\frac{\lambda_{1} \Lambda_{4}\left[\left(t-\eta_{2}\right) \Lambda_{0} \Lambda_{5}-t^{2}-\Lambda_{6}\right]}{\Gamma(\omega)} \int_{0}^{1} \int_{0}^{s}(s-\tau)^{\omega-1} \vartheta(\tau) \mathrm{d} \tau \mathrm{d} s\right]
\end{aligned}
$$

for some $\vartheta \in(\mathcal{S E L})_{\mathcal{S}, k}$. One can easily check that $g_{0}$ is a solution for the hybrid inclusion problem (1)-(2) if and only if $g_{0}$ is a fixed point of the operator $\mathcal{G}$. Define the maps $\Phi_{1}$ : $\mathcal{X} \rightarrow \mathcal{X}$ by $\left(\Phi_{1} k\right)(t)=\xi\left(t, k(t), \int_{0}^{1} k(s) \mathrm{d} s\right)$ and $\Phi_{2}: \mathcal{X} \rightarrow \mathcal{P}(\mathcal{X})$ by

$$
\left(\Phi_{2} k\right)(t)=\{\zeta \in \mathcal{X}: \zeta(t)=b(t) \text { for } t \in[0,1]\},
$$


where

$$
\begin{aligned}
b(t)= & \frac{1}{\Gamma(\omega)} \int_{0}^{t}(t-s)^{\omega-1} \vartheta(s) \mathrm{d} s \\
& +\frac{\left[\left(\Lambda_{3} \Lambda_{7}-\eta_{2} \Lambda_{5}\right)+t\left(\lambda_{1} \Lambda_{0} \Lambda_{3}-1\right) \Lambda_{5}-t^{2} \lambda_{1} \Lambda_{3}\right] \sum_{j=1}^{r} b_{j}}{\Gamma(\omega-1)} \int_{0}^{\eta_{1}}\left(\eta_{1}-s\right)^{\omega-2} \vartheta(s) \mathrm{d} s \\
& +\frac{1+\left(t \Lambda_{8}+\eta_{2}\right) \Lambda_{5}+\left(\Lambda_{3}+\Lambda_{4}\right)\left(t^{2} \lambda_{1}-\Lambda_{7}\right)}{\Gamma(\omega)} \int_{0}^{\eta_{2}}\left(\eta_{2}-s\right)^{\omega-1} \vartheta(s) \mathrm{d} s \\
& +\frac{\lambda_{2}\left[\left(1+t \Lambda_{5}\right) \Lambda_{8}+t^{2} \lambda_{1}\left(\Lambda_{3}+\Lambda_{4}\right)\right] \sum_{j=1}^{r} b_{j}}{\lambda_{1} \Gamma(\omega-2)} \int_{0}^{1}(1-s)^{\omega-3} \vartheta(s) \mathrm{d} s \\
& +\frac{\lambda_{1} \Lambda_{4}\left[\left(t-\eta_{2}\right) \Lambda_{0} \Lambda_{5}-t^{2}-\Lambda_{6}\right]}{\Gamma(\omega)} \int_{0}^{1} \int_{0}^{s}(s-\tau)^{\omega-1} \vartheta(\tau) \mathrm{d} \tau \mathrm{d} s
\end{aligned}
$$

for some $\vartheta \in(\mathcal{S E} \mathcal{L})_{\mathcal{S}, k}$. Then we obtain $\mathcal{G}(k)=\Phi_{1} k \Phi_{2} k$. We prove that $\Phi_{1}$ and $\Phi_{2}$ satisfy the assumptions of Theorem 2. We first show that the operator $\Phi_{1}$ is Lipschitz. Let $k_{1}, k_{2} \in$ $\mathcal{X}$. Assumption $(\mathcal{C} 1)$ implies that

$$
\begin{aligned}
\left|\left(\Phi_{1} k_{1}\right)(t)-\left(\Phi_{1} k_{2}\right)(t)\right| & =\left|\xi\left(t, k_{1}(t), \int_{0}^{1} k_{1}(s) \mathrm{d} s\right)-\xi\left(t, k_{2}(t), \int_{0}^{1} k_{2}(s) \mathrm{d} s\right)\right| \\
& \leq \theta(t)\left(\left|k_{1}(t)-k_{2}(t)\right|+\left|k_{1}(t)-k_{2}(t)\right|\right) \\
& =2 \theta(t)\left|k_{1}(t)-k_{2}(t)\right|
\end{aligned}
$$

for all $t \in[0,1]$. Hence, we get $\left\|\Phi_{1} k_{1}-\Phi_{1} k_{2}\right\|_{\mathcal{X}} \leq 2 \theta^{*}\left\|k_{1}-k_{2}\right\|_{\mathcal{X}}$ for all $k_{1}, k_{2} \in \mathcal{X}$. This means that the operator $\Phi_{1}$ is Lipschitz with constant $2 \theta^{*}$. Now, we claim that the setvalued map $\Phi_{2}$ has convex values. Let $k_{1}, k_{2} \in \Phi_{2} k$. Choose $\vartheta_{1}, \vartheta_{2}$ in $(\mathcal{S E} \mathcal{L})_{\mathcal{S}, k}$ such that

$$
\begin{aligned}
k_{i}(t)= & \frac{1}{\Gamma(\omega)} \int_{0}^{t}(t-s)^{\omega-1} \vartheta_{i}(s) \mathrm{d} s \\
& +\frac{\left[\left(\Lambda_{3} \Lambda_{7}-\eta_{2} \Lambda_{5}\right)+t\left(\lambda_{1} \Lambda_{0} \Lambda_{3}-1\right) \Lambda_{5}-t^{2} \lambda_{1} \Lambda_{3}\right] \sum_{j=1}^{r} b_{j}}{\Gamma(\omega-1)} \\
& \times \int_{0}^{\eta_{1}}\left(\eta_{1}-s\right)^{\omega-2} \vartheta_{i}(s) \mathrm{d} s \\
& +\frac{1+\left(t \Lambda_{8}+\eta_{2}\right) \Lambda_{5}+\left(\Lambda_{3}+\Lambda_{4}\right)\left(t^{2} \lambda_{1}-\Lambda_{7}\right)}{\Gamma(\omega)} \int_{0}^{\eta_{2}}\left(\eta_{2}-s\right)^{\omega-1} \vartheta_{i}(s) \mathrm{d} s \\
& +\frac{\lambda_{2}\left[\left(1+t \Lambda_{5}\right) \Lambda_{8}+t^{2} \lambda_{1}\left(\Lambda_{3}+\Lambda_{4}\right)\right] \sum_{j=1}^{r} b_{j}}{\lambda_{1} \Gamma(\omega-2)} \int_{0}^{1}(1-s)^{\omega-3} \vartheta_{i}(s) \mathrm{d} s \\
& +\frac{\lambda_{1} \Lambda_{4}\left[\left(t-\eta_{2}\right) \Lambda_{0} \Lambda_{5}-t^{2}-\Lambda_{6}\right]}{\Gamma(\omega)} \int_{0}^{1} \int_{0}^{s}(s-\tau)^{\omega-1} \vartheta_{i}(\tau) \mathrm{d} \tau \mathrm{d} s, \quad(i=1,2)
\end{aligned}
$$

for almost all $t \in[0,1]$. Let $\lambda \in(0,1)$. Then we have

$$
\begin{aligned}
\lambda k_{1}(t)+(1-\lambda) k_{2}(t)= & \frac{1}{\Gamma(\omega)} \int_{0}^{t}(t-s)^{\omega-1}\left[\lambda \vartheta_{1}(s)+(1-\lambda) \vartheta_{2}(s)\right] \mathrm{d} s \\
& +\frac{\left[\left(\Lambda_{3} \Lambda_{7}-\eta_{2} \Lambda_{5}\right)+t\left(\lambda_{1} \Lambda_{0} \Lambda_{3}-1\right) \Lambda_{5}-t^{2} \lambda_{1} \Lambda_{3}\right] \sum_{j=1}^{r} b_{j}}{\Gamma(\omega-1)}
\end{aligned}
$$




$$
\begin{aligned}
& \times \int_{0}^{\eta_{1}}\left(\eta_{1}-s\right)^{\omega-2}\left[\lambda \vartheta_{1}(s)+(1-\lambda) \vartheta_{2}(s)\right] \mathrm{d} s \\
& +\frac{1+\left(t \Lambda_{8}+\eta_{2}\right) \Lambda_{5}+\left(\Lambda_{3}+\Lambda_{4}\right)\left(t^{2} \lambda_{1}-\Lambda_{7}\right)}{\Gamma(\omega)} \\
& \times \int_{0}^{\eta_{2}}\left(\eta_{2}-s\right)^{\omega-1}\left[\lambda \vartheta_{1}(s)+(1-\lambda) \vartheta_{2}(s)\right] \mathrm{d} s \\
& +\frac{\lambda_{2}\left[\left(1+t \Lambda_{5}\right) \Lambda_{8}+t^{2} \lambda_{1}\left(\Lambda_{3}+\Lambda_{4}\right)\right] \sum_{j=1}^{r} b_{j}}{\lambda_{1} \Gamma(\omega-2)} \\
& \times \int_{0}^{1}(1-s)^{\omega-3}\left[\lambda \vartheta_{1}(s)+(1-\lambda) \vartheta_{2}(s)\right] \mathrm{d} s \\
& +\frac{\lambda_{1} \Lambda_{4}\left[\left(t-\eta_{2}\right) \Lambda_{0} \Lambda_{5}-t^{2}-\Lambda_{6}\right]}{\Gamma(\omega)} \\
& \times \int_{0}^{1} \int_{0}^{s}(s-\tau)^{\omega-1}\left[\lambda \vartheta_{1}(\tau)+(1-\lambda) \vartheta_{2}(\tau)\right] \mathrm{d} \tau \mathrm{d} s
\end{aligned}
$$

for almost all $t \in[0,1]$. Since $\mathcal{S}$ has convex values, $(\mathcal{S E} \mathcal{L})_{\mathcal{S}, k}$ is convex-valued. This gives that $\lambda \vartheta_{1}(t)+(1-\lambda) \vartheta_{2}(t) \in(\mathcal{S E} \mathcal{L})_{\mathcal{S}, k}$ for all $t \in[0,1]$, and so $\Phi_{2} k$ is a convex set for all $k \in \mathcal{X}$.

Now, we prove that the operator $\Phi_{2}$ is completely continuous. We have to prove the equi-continuity and uniform boundedness of the set $\Phi_{2}(\mathcal{X})$. First, we show that $\Phi_{2}$ maps all bounded sets into bounded subsets of $\mathcal{X}$. For a positive number $\varepsilon^{*} \in \mathbb{R}$, consider the bounded ball $\mathcal{V}_{\varepsilon^{*}}=\left\{k \in \mathcal{X}:\|k\|_{\mathcal{X}} \leq \varepsilon^{*}\right\}$. For every $k \in \mathcal{V}_{\varepsilon^{*}}$ and $\zeta \in \Phi_{2} k$, there exists a function $\vartheta \in(\mathcal{S E} \mathcal{L})_{\mathcal{S}, k}$ so that

$$
\begin{aligned}
\zeta(t)= & \frac{1}{\Gamma(\omega)} \int_{0}^{t}(t-s)^{\omega-1} \vartheta(s) \mathrm{d} s \\
& +\frac{\left[\left(\Lambda_{3} \Lambda_{7}-\eta_{2} \Lambda_{5}\right)+t\left(\lambda_{1} \Lambda_{0} \Lambda_{3}-1\right) \Lambda_{5}-t^{2} \lambda_{1} \Lambda_{3}\right] \sum_{j=1}^{r} b_{j}}{\Gamma(\omega-1)} \\
& \times \int_{0}^{\eta_{1}}\left(\eta_{1}-s\right)^{\omega-2} \vartheta(s) \mathrm{d} s \\
& +\frac{1+\left(t \Lambda_{8}+\eta_{2}\right) \Lambda_{5}+\left(\Lambda_{3}+\Lambda_{4}\right)\left(t^{2} \lambda_{1}-\Lambda_{7}\right)}{\Gamma(\omega)} \int_{0}^{\eta_{2}}\left(\eta_{2}-s\right)^{\omega-1} \vartheta(s) \mathrm{d} s \\
& +\frac{\lambda_{2}\left[\left(1+t \Lambda_{5}\right) \Lambda_{8}+t^{2} \lambda_{1}\left(\Lambda_{3}+\Lambda_{4}\right)\right] \sum_{j=1}^{r} b_{j}}{\lambda_{1} \Gamma(\omega-2)} \int_{0}^{1}(1-s)^{\omega-3} \vartheta(s) \mathrm{d} s \\
& +\frac{\lambda_{1} \Lambda_{4}\left[\left(t-\eta_{2}\right) \Lambda_{0} \Lambda_{5}-t^{2}-\Lambda_{6}\right]}{\Gamma(\omega)} \int_{0}^{1} \int_{0}^{s}(s-\tau)^{\omega-1} \vartheta(\tau) \mathrm{d} \tau \mathrm{d} s
\end{aligned}
$$

for all $t \in[0,1]$. Then we have

$$
\begin{aligned}
|\zeta(t)| \leq & \frac{1}{\Gamma(\omega)} \int_{0}^{t}(t-s)^{\omega-1}|\vartheta(s)| \mathrm{d} s \\
& +\frac{\left|\left(\Lambda_{3} \Lambda_{7}-\eta_{2} \Lambda_{5}\right)+t\left(\lambda_{1} \Lambda_{0} \Lambda_{3}-1\right) \Lambda_{5}-t^{2} \lambda_{1} \Lambda_{3}\right| \sum_{j=1}^{r} b_{j}}{\Gamma(\omega-1)} \\
& \times \int_{0}^{\eta_{1}}\left(\eta_{1}-s\right)^{\omega-2}|\vartheta(s)| \mathrm{d} s
\end{aligned}
$$




$$
\begin{aligned}
& +\frac{\left|1+\left(t \Lambda_{8}+\eta_{2}\right) \Lambda_{5}+\left(\Lambda_{3}+\Lambda_{4}\right)\left(t^{2} \lambda_{1}-\Lambda_{7}\right)\right|}{\Gamma(\omega)} \int_{0}^{\eta_{2}}\left(\eta_{2}-s\right)^{\omega-1}|\vartheta(s)| \mathrm{d} s \\
& +\frac{\lambda_{2}\left|\left(1+t \Lambda_{5}\right) \Lambda_{8}+t^{2} \lambda_{1}\left(\Lambda_{3}+\Lambda_{4}\right)\right| \sum_{j=1}^{r} b_{j}}{\lambda_{1} \Gamma(\omega-2)} \\
& \times \int_{0}^{1}(1-s)^{\omega-3}|\vartheta(s)| \mathrm{d} s \\
& +\frac{\lambda_{1}\left|\Lambda_{4}\left[\left(t-\eta_{2}\right) \Lambda_{0} \Lambda_{5}-t^{2}-\Lambda_{6}\right]\right|}{\Gamma(\omega)} \\
& \times \int_{0}^{1} \int_{0}^{s}(s-\tau)^{\omega-1}|\vartheta(\tau)| \mathrm{d} \tau \mathrm{d} s \leq \frac{1}{\Gamma(\omega)} \int_{0}^{t}(t-s)^{\omega-1} q(s) \mathrm{d} s \\
& +\frac{\left|\left(\Lambda_{3} \Lambda_{7}-\eta_{2} \Lambda_{5}\right)+t\left(\lambda_{1} \Lambda_{0} \Lambda_{3}-1\right) \Lambda_{5}-t^{2} \lambda_{1} \Lambda_{3}\right| \sum_{j=1}^{r} b_{j}}{\Gamma(\omega-1)} \\
& \times \int_{0}^{\eta_{1}}\left(\eta_{1}-s\right)^{\omega-2} q(s) \mathrm{d} s \\
& +\frac{\left|1+\left(t \Lambda_{8}+\eta_{2}\right) \Lambda_{5}+\left(\Lambda_{3}+\Lambda_{4}\right)\left(t^{2} \lambda_{1}-\Lambda_{7}\right)\right|}{\Gamma(\omega)} \int_{0}^{\eta_{2}}\left(\eta_{2}-s\right)^{\omega-1} q(s) \mathrm{d} s \\
& +\frac{\lambda_{2}\left|\left(1+t \Lambda_{5}\right) \Lambda_{8}+t^{2} \lambda_{1}\left(\Lambda_{3}+\Lambda_{4}\right)\right| \sum_{j=1}^{r} b_{j}}{\lambda_{1} \Gamma(\omega-2)} \int_{0}^{1}(1-s)^{\omega-3} q(s) \mathrm{d} s \\
& +\frac{\lambda_{1}\left|\Lambda_{4}\left[\left(t-\eta_{2}\right) \Lambda_{0} \Lambda_{5}-t^{2}-\Lambda_{6}\right]\right|}{\Gamma(\omega)} \int_{0}^{1} \int_{0}^{s}(s-\tau)^{\omega-1} q(\tau) \mathrm{d} \tau \mathrm{d} s \\
& \leq\left[\frac{1}{\Gamma(\omega+1)}\right. \\
& +\frac{\left[\left|\Lambda_{3} \Lambda_{7}\right|+\left|\eta_{2} \Lambda_{5}\right|+\left(\left|\lambda_{1} \Lambda_{0} \Lambda_{3}\right|+1\right)\left|\Lambda_{5}\right|+\left|\lambda_{1} \Lambda_{3}\right|\right] \eta_{1}^{\omega-1} \sum_{j=1}^{r} b_{j}}{\Gamma(\omega)} \\
& +\frac{\left[1+\left|\left(\Lambda_{8}+\eta_{2}\right) \Lambda_{5}\right|+\left|\left(\Lambda_{3}+\Lambda_{4}\right)\right|\left(\lambda_{1}+\left|\Lambda_{7}\right|\right)\right] \eta_{2}^{\omega}}{\Gamma(\omega+1)} \\
& +\frac{\lambda_{2}\left[\left|\left(1+\Lambda_{5}\right) \Lambda_{8}\right|+\lambda_{1}\left|\Lambda_{3}+\Lambda_{4}\right|\right] \sum_{j=1}^{r} b_{j}}{\lambda_{1} \Gamma(\omega-1)} \\
& \left.+\frac{\lambda_{1}\left|\Lambda_{4}\right|\left[\left(1+\eta_{2}\right)\left|\Lambda_{0} \Lambda_{5}\right|+\left|\Lambda_{6}\right|+1\right]}{\Gamma(\omega+2)}\right]\|q\|_{\mathcal{L}^{1}} \\
& =M\|q\|_{\mathcal{L}^{1}},
\end{aligned}
$$

where $M$ is given in (9). Thus, $\|\zeta\| \leq M\|q\|_{\mathcal{L}^{1}}$ and this shows that the set $\Phi_{2}(\mathcal{X})$ is uniformly bounded. Next, we prove that the operator $\Phi_{2}$ maps bounded sets into equicontinuous sets. Let $k \in V_{\mathcal{E}^{*}}$ and $\zeta \in \Phi_{2} k$. Choose $\vartheta \in(\mathcal{S E L})_{\mathcal{S}, k}$ such that

$$
\begin{aligned}
\zeta(t)= & \frac{1}{\Gamma(\omega)} \int_{0}^{t}(t-s)^{\omega-1} \vartheta(s) \mathrm{d} s \\
& +\frac{\left[\left(\Lambda_{3} \Lambda_{7}-\eta_{2} \Lambda_{5}\right)+t\left(\lambda_{1} \Lambda_{0} \Lambda_{3}-1\right) \Lambda_{5}-t^{2} \lambda_{1} \Lambda_{3}\right] \sum_{j=1}^{r} b_{j}}{\Gamma(\omega-1)} \\
& \times \int_{0}^{\eta_{1}}\left(\eta_{1}-s\right)^{\omega-2} \vartheta(s) \mathrm{d} s
\end{aligned}
$$




$$
\begin{aligned}
& +\frac{1+\left(t \Lambda_{8}+\eta_{2}\right) \Lambda_{5}+\left(\Lambda_{3}+\Lambda_{4}\right)\left(t^{2} \lambda_{1}-\Lambda_{7}\right)}{\Gamma(\omega)} \int_{0}^{\eta_{2}}\left(\eta_{2}-s\right)^{\omega-1} \vartheta(s) \mathrm{d} s \\
& +\frac{\lambda_{2}\left[\left(1+t \Lambda_{5}\right) \Lambda_{8}+t^{2} \lambda_{1}\left(\Lambda_{3}+\Lambda_{4}\right)\right] \sum_{j=1}^{r} b_{j}}{\lambda_{1} \Gamma(\omega-2)} \int_{0}^{1}(1-s)^{\omega-3} \vartheta(s) \mathrm{d} s \\
& +\frac{\lambda_{1} \Lambda_{4}\left[\left(t-\eta_{2}\right) \Lambda_{0} \Lambda_{5}-t^{2}-\Lambda_{6}\right]}{\Gamma(\omega)} \int_{0}^{1} \int_{0}^{s}(s-\tau)^{\omega-1} \vartheta(\tau) \mathrm{d} \tau \mathrm{d} s
\end{aligned}
$$

for all $t \in[0,1]$. Let $t_{1}, t_{2} \in[0,1]$ with $t_{1}<t_{2}$. Then we have

$$
\begin{aligned}
\left|\zeta\left(t_{2}\right)-\zeta\left(t_{1}\right)\right| \leq & \frac{1}{\Gamma(\omega)} \int_{0}^{t_{1}}\left[\left(t_{2}-s\right)^{\omega-1}-\left(t_{1}-s\right)^{\omega-1}\right]|\vartheta(s)| \mathrm{d} s \\
& +\frac{1}{\Gamma(\omega)} \int_{t_{1}}^{t_{2}}\left(t_{2}-s\right)^{\omega-1}|\vartheta(s)| \mathrm{d} s \\
& +\frac{\left[\left|\left(t_{2}-t_{1}\right)\left(\lambda_{1} \Lambda_{0} \Lambda_{3}-1\right) \Lambda_{5}\right|+\left|\left(t_{2}^{2}-t_{1}^{2}\right) \lambda_{1} \Lambda_{3}\right|\right] \sum_{j=1}^{r} b_{j}}{\Gamma(\omega-1)} \\
& \times \int_{0}^{\eta_{1}}\left(\eta_{1}-s\right)^{\omega-2}|\vartheta(s)| \mathrm{d} s \\
& +\frac{\left|\left(t_{2}-t_{1}\right) \Lambda_{8} \Lambda_{5}\right|+\left|\lambda_{1}\left(\Lambda_{3}+\Lambda_{4}\right)\left(t_{2}^{2}-t_{1}^{2}\right)\right|}{\Gamma(\omega)} \int_{0}^{\eta_{2}}\left(\eta_{2}-s\right)^{\omega-1}|\vartheta(s)| \mathrm{d} s \\
& +\frac{\lambda_{2}\left[\left|\left(t_{2}-t_{1}\right) \Lambda_{5} \Lambda_{8}\right|+\left|\left(t_{2}^{2}-t_{1}^{2}\right) \lambda_{1}\left(\Lambda_{3}+\Lambda_{4}\right)\right|\right] \sum_{j=1}^{r} b_{j}}{\lambda_{1} \Gamma(\omega-2)} \\
& \times \int_{0}^{1}(1-s)^{\omega-3}|\vartheta(s)| \mathrm{d} s \\
& +\frac{\lambda_{1}\left|\Lambda_{4}\right|\left[\left|\left(t_{2}-t_{1}\right) \Lambda_{0} \Lambda_{5}\right|+\left|t_{2}^{2}-t_{1}^{2}\right|\right]}{\Gamma(\omega)} \int_{0}^{1} \int_{0}^{s}(s-\tau)^{\omega-1}|\vartheta(\tau)| \mathrm{d} \tau \mathrm{d} s \\
\leq & \frac{1}{\Gamma(\omega)} \int_{0}^{t_{1}}\left[\left(t_{2}-s\right)^{\omega-1}-\left(t_{1}-s\right)^{\omega-1}\right] q(s) \mathrm{d} s+\frac{1}{\Gamma(\omega)} \\
& \times \int_{t_{1}}^{t_{2}}\left(t_{2}-s\right)^{\omega-1} q(s) \mathrm{d} s \\
& +\frac{\left[\left|\left(t_{2}-t_{1}\right)\left(\lambda_{1} \Lambda_{0} \Lambda_{3}-1\right) \Lambda_{5}\right|+\left|\left(t_{2}^{2}-t_{1}^{2}\right) \lambda_{1} \Lambda_{3}\right|\right] \sum_{j=1}^{r} b_{j}}{\Gamma(\omega-1)} \\
& +\frac{\lambda_{2}\left[\left|\left(t_{2}-t_{1}\right) \Lambda_{5} \Lambda_{8}\right|+\left|\left(t_{2}^{2}-t_{1}^{2}\right) \lambda_{1}\left(\Lambda_{3}+\Lambda_{4}\right)\right|\right] \sum_{j=1}^{r} b_{j}}{\eta_{1} \Gamma(\omega-2)} \\
& \times \int_{0}^{\eta_{1}}\left(\eta_{1}-s\right)^{\omega-2} q(s) \mathrm{d} s \\
& \left|\left(t_{2}-t_{1}\right) \Lambda_{8} \Lambda_{5}\right|+\left|\lambda_{1}\left(\Lambda_{3}+\Lambda_{4}\right)\left(t_{2}^{2}-t_{1}^{2}\right)\right| \\
& \\
& \\
& \\
&
\end{aligned}
$$




$$
\begin{aligned}
& +\frac{\lambda_{1}\left|\Lambda_{4}\right|\left[\left|\left(t_{2}-t_{1}\right) \Lambda_{0} \Lambda_{5}\right|+\left|t_{2}^{2}-t_{1}^{2}\right|\right]}{\Gamma(\omega)} \\
& \times \int_{0}^{1} \int_{0}^{s}(s-\tau)^{\omega-1} q(\tau) \mathrm{d} \tau \mathrm{d} s .
\end{aligned}
$$

Note that the right-hand side tends to zero independently of $k \in \mathcal{V}_{\varepsilon^{*}}$ as $t_{2} \rightarrow t_{1}$. By using the Arzela-Ascoli theorem, the complete continuity of $\Phi_{2}: C([0,1], \mathbb{R}) \rightarrow \mathcal{P}(C([0,1], \mathbb{R}))$ is deduced. Now, we show that $\Phi_{2}$ has a closed graph, and this follows the upper semicontinuity of the operator $\Phi_{2}$. Assume that $k_{n} \in \mathcal{V}_{\varepsilon^{*}}$ and $\zeta_{n} \in \Phi_{2} k_{n}$ with $k_{n} \rightarrow k^{*}$ and $\zeta_{n} \rightarrow$ $\zeta^{*}$. We claim that $\zeta^{*} \in \Phi_{2} k^{*}$. For every $n \geq 1$ and $\zeta_{n} \in \Phi_{2} k_{n}$, choose $\vartheta_{n} \in(\mathcal{S E} \mathcal{L})_{\mathcal{S}, k_{n}}$ such that

$$
\begin{aligned}
\zeta_{n}(t)= & \frac{1}{\Gamma(\omega)} \int_{0}^{t}(t-s)^{\omega-1} \vartheta_{n}(s) \mathrm{d} s \\
& +\frac{\left[\left(\Lambda_{3} \Lambda_{7}-\eta_{2} \Lambda_{5}\right)+t\left(\lambda_{1} \Lambda_{0} \Lambda_{3}-1\right) \Lambda_{5}-t^{2} \lambda_{1} \Lambda_{3}\right] \sum_{j=1}^{r} b_{j}}{\Gamma(\omega-1)} \\
& \times \int_{0}^{\eta_{1}}\left(\eta_{1}-s\right)^{\omega-2} \vartheta_{n}(s) \mathrm{d} s \\
& +\frac{1+\left(t \Lambda_{8}+\eta_{2}\right) \Lambda_{5}+\left(\Lambda_{3}+\Lambda_{4}\right)\left(t^{2} \lambda_{1}-\Lambda_{7}\right)}{\Gamma(\omega)} \int_{0}^{\eta_{2}}\left(\eta_{2}-s\right)^{\omega-1} \vartheta_{n}(s) \mathrm{d} s \\
& +\frac{\lambda_{2}\left[\left(1+t \Lambda_{5}\right) \Lambda_{8}+t^{2} \lambda_{1}\left(\Lambda_{3}+\Lambda_{4}\right)\right] \sum_{j=1}^{r} b_{j}}{\lambda_{1} \Gamma(\omega-2)} \int_{0}^{1}(1-s)^{\omega-3} \vartheta_{n}(s) \mathrm{d} s \\
& +\frac{\lambda_{1} \Lambda_{4}\left[\left(t-\eta_{2}\right) \Lambda_{0} \Lambda_{5}-t^{2}-\Lambda_{6}\right]}{\Gamma(\omega)} \int_{0}^{1} \int_{0}^{s}(s-\tau)^{\omega-1} \vartheta_{n}(\tau) \mathrm{d} \tau \mathrm{d} s
\end{aligned}
$$

for all $t \in[0,1]$. It is sufficient to show that there exists a function $\vartheta^{*} \in(\mathcal{S E L})_{\mathcal{S}, k^{*}}$ such that

$$
\begin{aligned}
\zeta^{*}(t)= & \frac{1}{\Gamma(\omega)} \int_{0}^{t}(t-s)^{\omega-1} \vartheta^{*}(s) \mathrm{d} s \\
& +\frac{\left[\left(\Lambda_{3} \Lambda_{7}-\eta_{2} \Lambda_{5}\right)+t\left(\lambda_{1} \Lambda_{0} \Lambda_{3}-1\right) \Lambda_{5}-t^{2} \lambda_{1} \Lambda_{3}\right] \sum_{j=1}^{r} b_{j}}{\Gamma(\omega-1)} \\
& \times \int_{0}^{\eta_{1}}\left(\eta_{1}-s\right)^{\omega-2} \vartheta^{*}(s) \mathrm{d} s \\
& +\frac{1+\left(t \Lambda_{8}+\eta_{2}\right) \Lambda_{5}+\left(\Lambda_{3}+\Lambda_{4}\right)\left(t^{2} \lambda_{1}-\Lambda_{7}\right)}{\Gamma(\omega)} \int_{0}^{\eta_{2}}\left(\eta_{2}-s\right)^{\omega-1} \vartheta^{*}(s) \mathrm{d} s \\
& +\frac{\lambda_{2}\left[\left(1+t \Lambda_{5}\right) \Lambda_{8}+t^{2} \lambda_{1}\left(\Lambda_{3}+\Lambda_{4}\right)\right] \sum_{j=1}^{r} b_{j}}{\lambda_{1} \Gamma(\omega-2)} \int_{0}^{1}(1-s)^{\omega-3} \vartheta^{*}(s) \mathrm{d} s \\
& +\frac{\lambda_{1} \Lambda_{4}\left[\left(t-\eta_{2}\right) \Lambda_{0} \Lambda_{5}-t^{2}-\Lambda_{6}\right]}{\Gamma(\omega)} \int_{0}^{1} \int_{0}^{s}(s-\tau)^{\omega-1} \vartheta^{*}(\tau) \mathrm{d} \tau \mathrm{d} s
\end{aligned}
$$

for all $t \in[0,1]$. Define the continuous linear operator $\Xi: \mathcal{L}^{1}([0,1], \mathbb{R}) \rightarrow \mathcal{X}$ by

$$
\begin{aligned}
\Xi(\vartheta)(t)= & k(t)=\frac{1}{\Gamma(\omega)} \int_{0}^{t}(t-s)^{\omega-1} \vartheta(s) \mathrm{d} s \\
& +\frac{\left[\left(\Lambda_{3} \Lambda_{7}-\eta_{2} \Lambda_{5}\right)+t\left(\lambda_{1} \Lambda_{0} \Lambda_{3}-1\right) \Lambda_{5}-t^{2} \lambda_{1} \Lambda_{3}\right] \sum_{j=1}^{r} b_{j}}{\Gamma(\omega-1)}
\end{aligned}
$$




$$
\begin{aligned}
& \times \int_{0}^{\eta_{1}}\left(\eta_{1}-s\right)^{\omega-2} \vartheta(s) \mathrm{d} s \\
& +\frac{1+\left(t \Lambda_{8}+\eta_{2}\right) \Lambda_{5}+\left(\Lambda_{3}+\Lambda_{4}\right)\left(t^{2} \lambda_{1}-\Lambda_{7}\right)}{\Gamma(\omega)} \int_{0}^{\eta_{2}}\left(\eta_{2}-s\right)^{\omega-1} \vartheta(s) \mathrm{d} s \\
& +\frac{\lambda_{2}\left[\left(1+t \Lambda_{5}\right) \Lambda_{8}+t^{2} \lambda_{1}\left(\Lambda_{3}+\Lambda_{4}\right)\right] \sum_{j=1}^{r} b_{j}}{\lambda_{1} \Gamma(\omega-2)} \int_{0}^{1}(1-s)^{\omega-3} \vartheta(s) \mathrm{d} s \\
& +\frac{\lambda_{1} \Lambda_{4}\left[\left(t-\eta_{2}\right) \Lambda_{0} \Lambda_{5}-t^{2}-\Lambda_{6}\right]}{\Gamma(\omega)} \int_{0}^{1} \int_{0}^{s}(s-\tau)^{\omega-1} \vartheta(\tau) \mathrm{d} \tau \mathrm{d} s
\end{aligned}
$$

for all $t \in[0,1]$, where $\mathcal{X}=C([0,1], \mathbb{R})$. Hence,

$$
\begin{aligned}
\left\|\zeta_{n}(t)-\zeta^{*}(t)\right\|= & \| \frac{1}{\Gamma(\omega)} \int_{0}^{t}(t-s)^{\omega-1}\left(\vartheta_{n}(s)-\vartheta^{*}(s)\right) \mathrm{d} s \\
& +\frac{\left[\left(\Lambda_{3} \Lambda_{7}-\eta_{2} \Lambda_{5}\right)+t\left(\lambda_{1} \Lambda_{0} \Lambda_{3}-1\right) \Lambda_{5}-t^{2} \lambda_{1} \Lambda_{3}\right] \sum_{j=1}^{r} b_{j}}{\Gamma(\omega-1)} \\
& \times \int_{0}^{\eta_{1}}\left(\eta_{1}-s\right)^{\omega-2}\left(\vartheta_{n}(s)-\vartheta^{*}(s)\right) \mathrm{d} s \\
& +\frac{1+\left(t \Lambda_{8}+\eta_{2}\right) \Lambda_{5}+\left(\Lambda_{3}+\Lambda_{4}\right)\left(t^{2} \lambda_{1}-\Lambda_{7}\right)}{\Gamma(\omega)} \\
& \times \int_{0}^{\eta_{2}}\left(\eta_{2}-s\right)^{\omega-1}\left(\vartheta_{n}(s)-\vartheta^{*}(s)\right) \mathrm{d} s \\
& +\frac{\lambda_{2}\left[\left(1+t \Lambda_{5}\right) \Lambda_{8}+t^{2} \lambda_{1}\left(\Lambda_{3}+\Lambda_{4}\right)\right] \sum_{j=1}^{r} b_{j}}{\lambda_{1} \Gamma(\omega-2)} \\
& \times \int_{0}^{1}(1-s)^{\omega-3}\left(\vartheta_{n}(s)-\vartheta^{*}(s)\right) \mathrm{d} s \\
& +\frac{\lambda_{1} \Lambda_{4}\left[\left(t-\eta_{2}\right) \Lambda_{0} \Lambda_{5}-t^{2}-\Lambda_{6}\right]}{\Gamma(\omega)} \\
& \times \int_{0}^{1} \int_{0}^{s}(s-\tau)^{\omega-1}\left(\vartheta_{n}(\tau)-\vartheta^{*}(\tau)\right) \mathrm{d} \tau \mathrm{d} s \| \\
\rightarrow & 0 .
\end{aligned}
$$

Hence, Theorem 1 implies that the operator $\Xi \circ(\mathcal{S E L})_{\mathcal{S}}$ has a closed graph. Since $\zeta_{n} \in$ $\Xi\left((\mathcal{S E L})_{\mathcal{S}, k_{n}}\right)$ and $k_{n} \rightarrow k^{*}$, there exists $\vartheta^{*} \in(\mathcal{S E L})_{\mathcal{S}, k^{*}}$ such that

$$
\begin{aligned}
\zeta^{*}(t)= & \frac{1}{\Gamma(\omega)} \int_{0}^{t}(t-s)^{\omega-1} \vartheta^{*}(s) \mathrm{d} s \\
& +\frac{\left[\left(\Lambda_{3} \Lambda_{7}-\eta_{2} \Lambda_{5}\right)+t\left(\lambda_{1} \Lambda_{0} \Lambda_{3}-1\right) \Lambda_{5}-t^{2} \lambda_{1} \Lambda_{3}\right] \sum_{j=1}^{r} b_{j}}{\Gamma(\omega-1)} \\
& \times \int_{0}^{\eta_{1}}\left(\eta_{1}-s\right)^{\omega-2} \vartheta^{*}(s) \mathrm{d} s \\
& +\frac{1+\left(t \Lambda_{8}+\eta_{2}\right) \Lambda_{5}+\left(\Lambda_{3}+\Lambda_{4}\right)\left(t^{2} \lambda_{1}-\Lambda_{7}\right)}{\Gamma(\omega)} \int_{0}^{\eta_{2}}\left(\eta_{2}-s\right)^{\omega-1} \vartheta^{*}(s) \mathrm{d} s \\
& +\frac{\lambda_{2}\left[\left(1+t \Lambda_{5}\right) \Lambda_{8}+t^{2} \lambda_{1}\left(\Lambda_{3}+\Lambda_{4}\right)\right] \sum_{j=1}^{r} b_{j}}{\lambda_{1} \Gamma(\omega-2)} \int_{0}^{1}(1-s)^{\omega-3} \vartheta^{*}(s) \mathrm{d} s
\end{aligned}
$$




$$
+\frac{\lambda_{1} \Lambda_{4}\left[\left(t-\eta_{2}\right) \Lambda_{0} \Lambda_{5}-t^{2}-\Lambda_{6}\right]}{\Gamma(\omega)} \int_{0}^{1} \int_{0}^{s}(s-\tau)^{\omega-1} \vartheta^{*}(\tau) \mathrm{d} \tau \mathrm{d} s
$$

for all $t \in[0,1]$. Hence, $\zeta^{*} \in \Phi_{2} k^{*}$ and so $\Phi_{2}$ has a closed graph. From this it follows that the operator $\Phi_{2}$ is upper semi-continuous. Since the operator $\Phi_{2}$ has compact values, $\Phi_{2}$ is a compact and upper semi-continuous operator. By using assumption $(\mathcal{C} 3)$, we have

$$
\begin{aligned}
\hat{\Delta}= & \left\|\Phi_{2}(\mathcal{X})\right\|=\sup _{t \in[0,1]}\left\{\left|\Phi_{2} k\right|: k \in \mathcal{X}\right\} \\
= & {\left[\frac{1}{\Gamma(\omega+1)}\right.} \\
& +\frac{\left[\left|\Lambda_{3} \Lambda_{7}\right|+\left|\eta_{2} \Lambda_{5}\right|+\left(\left|\lambda_{1} \Lambda_{0} \Lambda_{3}\right|+1\right)\left|\Lambda_{5}\right|+\left|\lambda_{1} \Lambda_{3}\right|\right] \eta_{1}^{\omega-1} \sum_{j=1}^{r} b_{j}}{\Gamma(\omega)} \\
& +\frac{\left[1+\left|\left(\Lambda_{8}+\eta_{2}\right) \Lambda_{5}\right|+\left|\left(\Lambda_{3}+\Lambda_{4}\right)\right|\left(\lambda_{1}+\left|\Lambda_{7}\right|\right)\right] \eta_{2}^{\omega}}{\Gamma(\omega+1)} \\
& +\frac{\lambda_{2}\left[\left|\left(1+\Lambda_{5}\right) \Lambda_{8}\right|+\lambda_{1}\left|\Lambda_{3}+\Lambda_{4}\right|\right] \sum_{j=1}^{r} b_{j}}{\lambda_{1} \Gamma(\omega-1)} \\
& \left.+\frac{\lambda_{1}\left|\Lambda_{4}\right|\left[\left(1+\eta_{2}\right)\left|\Lambda_{0} \Lambda_{5}\right|+\left|\Lambda_{6}\right|+1\right]}{\Gamma(\omega+2)}\right]\|q\|_{\mathcal{L}^{1}} \\
= & M\|q\|_{\mathcal{L}^{1}} .
\end{aligned}
$$

Put $l^{*}=2 \theta^{*}$. Then $\hat{\Delta} l^{*}<\frac{1}{2}$. Now, by using Theorem 2 for $\Phi_{2}$, we get that one of the conditions, (a) or (b), holds. We claim that condition (b) is impossible. By considering Theorem 2 and assumption $(\mathcal{C} 4)$, assume that $k$ is an arbitrary element of $\mathcal{O}^{*}$ with $\|k\|=\tilde{\rho}$. Then $\alpha_{0} k(t) \in \Phi_{1} k(t) \Phi_{2} k(t)$ for all $\alpha_{0}>1$. Choose the related function $\vartheta \in(\mathcal{S E} \mathcal{L})_{\mathcal{S}, k}$. Then, for each $\alpha_{0}>1$, we have

$$
\begin{aligned}
k(t)= & \frac{1}{\alpha_{0}} \xi\left(t, k(t), \int_{0}^{1} k(s) \mathrm{d} s\right)\left[\frac{1}{\Gamma(\omega)} \int_{0}^{t}(t-s)^{\omega-1} \vartheta(s) \mathrm{d} s\right. \\
& +\frac{\left[\left(\Lambda_{3} \Lambda_{7}-\eta_{2} \Lambda_{5}\right)+t\left(\lambda_{1} \Lambda_{0} \Lambda_{3}-1\right) \Lambda_{5}-t^{2} \lambda_{1} \Lambda_{3}\right] \sum_{j=1}^{r} b_{j}}{\Gamma(\omega-1)} \\
& \times \int_{0}^{\eta_{1}}\left(\eta_{1}-s\right)^{\omega-2} \vartheta(s) \mathrm{d} s \\
& +\frac{1+\left(t \Lambda_{8}+\eta_{2}\right) \Lambda_{5}+\left(\Lambda_{3}+\Lambda_{4}\right)\left(t^{2} \lambda_{1}-\Lambda_{7}\right)}{\Gamma(\omega)} \int_{0}^{\eta_{2}}\left(\eta_{2}-s\right)^{\omega-1} \vartheta(s) \mathrm{d} s \\
& +\frac{\lambda_{2}\left[\left(1+t \Lambda_{5}\right) \Lambda_{8}+t^{2} \lambda_{1}\left(\Lambda_{3}+\Lambda_{4}\right)\right] \sum_{j=1}^{r} b_{j}}{\lambda_{1} \Gamma(\omega-2)} \int_{0}^{1}(1-s)^{\omega-3} \vartheta(s) \mathrm{d} s \\
& \left.+\frac{\lambda_{1} \Lambda_{4}\left[\left(t-\eta_{2}\right) \Lambda_{0} \Lambda_{5}-t^{2}-\Lambda_{6}\right]}{\Gamma(\omega)} \int_{0}^{1} \int_{0}^{s}(s-\tau)^{\omega-1} \vartheta(\tau) \mathrm{d} \tau \mathrm{d} s\right]
\end{aligned}
$$

for all $t \in[0,1]$. Thus, one can write

$$
\begin{aligned}
|k(t)|= & \frac{1}{\alpha_{0}}\left|\xi\left(t, k(t), \int_{0}^{1} k(s) \mathrm{d} s\right)\right|\left[\frac{1}{\Gamma(\omega)} \int_{0}^{t}(t-s)^{\omega-1}|\vartheta(s)| \mathrm{d} s\right. \\
& +\frac{\left|\left(\Lambda_{3} \Lambda_{7}-\eta_{2} \Lambda_{5}\right)+t\left(\lambda_{1} \Lambda_{0} \Lambda_{3}-1\right) \Lambda_{5}-t^{2} \lambda_{1} \Lambda_{3}\right| \sum_{j=1}^{r} b_{j}}{\Gamma(\omega-1)}
\end{aligned}
$$




$$
\begin{aligned}
& \times \int_{0}^{\eta_{1}}\left(\eta_{1}-s\right)^{\omega-2}|\vartheta(s)| \mathrm{d} s \\
& +\frac{\left|1+\left(t \Lambda_{8}+\eta_{2}\right) \Lambda_{5}+\left(\Lambda_{3}+\Lambda_{4}\right)\left(t^{2} \lambda_{1}-\Lambda_{7}\right)\right|}{\Gamma(\omega)} \int_{0}^{\eta_{2}}\left(\eta_{2}-s\right)^{\omega-1}|\vartheta(s)| \mathrm{d} s \\
& +\frac{\lambda_{2}\left|\left(1+t \Lambda_{5}\right) \Lambda_{8}+t^{2} \lambda_{1}\left(\Lambda_{3}+\Lambda_{4}\right)\right| \sum_{j=1}^{r} b_{j}}{\lambda_{1} \Gamma(\omega-2)} \int_{0}^{1}(1-s)^{\omega-3}|\vartheta(s)| \mathrm{d} s \\
& \left.+\frac{\lambda_{1}\left|\Lambda_{4}\left[\left(t-\eta_{2}\right) \Lambda_{0} \Lambda_{5}-t^{2}-\Lambda_{6}\right]\right|}{\Gamma(\omega)} \int_{0}^{1} \int_{0}^{s}(s-\tau)^{\omega-1}|\vartheta(\tau)| \mathrm{d} \tau \mathrm{d} s\right] \\
& =\left[\left|\xi\left(t, k(t), \int_{0}^{1} k(s) \mathrm{d} s\right)-\xi(t, 0,0)\right|+|\xi(t, 0,0)|\right] \\
& \times\left[\frac{1}{\Gamma(\omega)} \int_{0}^{t}(t-s)^{\omega-1}|\vartheta(s)| \mathrm{d} s\right. \\
& +\frac{\left|\left(\Lambda_{3} \Lambda_{7}-\eta_{2} \Lambda_{5}\right)+t\left(\lambda_{1} \Lambda_{0} \Lambda_{3}-1\right) \Lambda_{5}-t^{2} \lambda_{1} \Lambda_{3}\right| \sum_{j=1}^{r} b_{j}}{\Gamma(\omega-1)} \\
& \times \int_{0}^{\eta_{1}}\left(\eta_{1}-s\right)^{\omega-2}|\vartheta(s)| \mathrm{d} s \\
& +\frac{\left|1+\left(t \Lambda_{8}+\eta_{2}\right) \Lambda_{5}+\left(\Lambda_{3}+\Lambda_{4}\right)\left(t^{2} \lambda_{1}-\Lambda_{7}\right)\right|}{\Gamma(\omega)} \int_{0}^{\eta_{2}}\left(\eta_{2}-s\right)^{\omega-1}|\vartheta(s)| \mathrm{d} s \\
& +\frac{\lambda_{2}\left|\left(1+t \Lambda_{5}\right) \Lambda_{8}+t^{2} \lambda_{1}\left(\Lambda_{3}+\Lambda_{4}\right)\right| \sum_{j=1}^{r} b_{j}}{\lambda_{1} \Gamma(\omega-2)} \int_{0}^{1}(1-s)^{\omega-3}|\vartheta(s)| \mathrm{d} s \\
& \left.+\frac{\lambda_{1}\left|\Lambda_{4}\left[\left(t-\eta_{2}\right) \Lambda_{0} \Lambda_{5}-t^{2}-\Lambda_{6}\right]\right|}{\Gamma(\omega)} \int_{0}^{1} \int_{0}^{s}(s-\tau)^{\omega-1}|\vartheta(\tau)| \mathrm{d} \tau \mathrm{d} s\right] \\
& \leq\left[2 \theta^{*}\|k\|+\xi^{*}\right]\left[\frac{1}{\Gamma(\omega)} \int_{0}^{t}(t-s)^{\omega-1} q(s) \mathrm{d} s\right. \\
& +\frac{\left|\left(\Lambda_{3} \Lambda_{7}-\eta_{2} \Lambda_{5}\right)+t\left(\lambda_{1} \Lambda_{0} \Lambda_{3}-1\right) \Lambda_{5}-t^{2} \lambda_{1} \Lambda_{3}\right| \sum_{j=1}^{r} b_{j}}{\Gamma(\omega-1)} \\
& \times \int_{0}^{\eta_{1}}\left(\eta_{1}-s\right)^{\omega-2} q(s) \mathrm{d} s \\
& +\frac{\left|1+\left(t \Lambda_{8}+\eta_{2}\right) \Lambda_{5}+\left(\Lambda_{3}+\Lambda_{4}\right)\left(t^{2} \lambda_{1}-\Lambda_{7}\right)\right|}{\Gamma(\omega)} \int_{0}^{\eta_{2}}\left(\eta_{2}-s\right)^{\omega-1} q(s) \mathrm{d} s \\
& +\frac{\lambda_{2}\left|\left(1+t \Lambda_{5}\right) \Lambda_{8}+t^{2} \lambda_{1}\left(\Lambda_{3}+\Lambda_{4}\right)\right| \sum_{j=1}^{r} b_{j}}{\lambda_{1} \Gamma(\omega-2)} \int_{0}^{1}(1-s)^{\omega-3} q(s) \mathrm{d} s \\
& \left.+\frac{\lambda_{1}\left|\Lambda_{4}\left[\left(t-\eta_{2}\right) \Lambda_{0} \Lambda_{5}-t^{2}-\Lambda_{6}\right]\right|}{\Gamma(\omega)} \int_{0}^{1} \int_{0}^{s}(s-\tau)^{\omega-1} q(\tau) \mathrm{d} \tau \mathrm{d} s\right] \\
& \leq\left[2 \theta^{*} \tilde{\rho}+\xi^{*}\right] M\|q\|_{\mathcal{L}^{1}}
\end{aligned}
$$

for all $t \in[0,1]$. Hence, we get $\tilde{\rho} \leq \frac{\xi^{*} M\|q\|_{\mathcal{L}^{1}}}{1-2 \theta^{*} M\|q\|_{\mathcal{L}^{1}}}$. Now, by using (8), we conclude that condition (b) of Theorem 2 is impossible. Thus, $k \in \Phi_{1} k \Phi_{2} k$. Hence, the operator $\mathcal{G}$ has a fixed point, and so the hybrid inclusion problem (1)-(2) has a solution.

Here, we provide an example to illustrate our main results. 
Example 1 Consider the fractional hybrid multi-term integro-differential inclusion

$$
\begin{aligned}
& { }^{C} \mathcal{D}_{0}^{2.71}\left(\frac{k(t)}{\frac{t|\cos k(t)|}{(1+|\cos k(t)|) 10,000}+0.0001 t \int_{0}^{1} k(s) \mathrm{d} s+0.0009}\right) \\
& \quad \in\left[-2,(t+1) \cos k(t)+2 \sin \left(\mathcal{I}_{0}^{0.03} k(t)\right)+\frac{7}{10} \sin ^{2}\left(\mathcal{I}_{0}^{0.05} k(t)\right)+\frac{8}{10}\right],
\end{aligned}
$$

with four-point sum and integral boundary value conditions

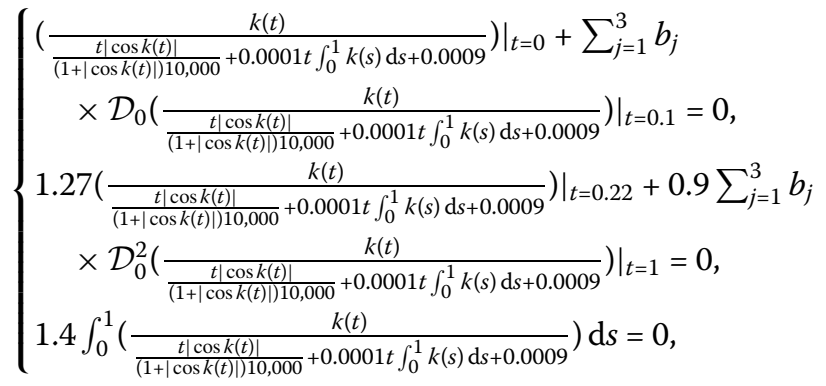

where $t \in[0,1], \omega=2.71, \eta_{1}=0.1, \eta_{2}=0.22, \lambda_{1}=1.27, \lambda_{2}=0.9, \lambda_{3}=1.4$, and $r=3$. Then we have $\sum_{j=1}^{3} b_{j}=0.24$ with $b_{1}=0.09, b_{2}=0.08$, and $b_{3}=0.07$. For $m=2$, put $\phi_{1}(k(t))=$ $\mathcal{I}_{0}^{0.03} k(t)$ and $\phi_{2}(k(t))=\mathcal{I}_{0}^{0.05} k(t)$, where $\varpi_{1}=0.03$ and $\varpi_{2}=0.05$. Consider the continuous $\operatorname{map} \xi:[0,1] \times \mathbb{R} \times \mathbb{R} \rightarrow \mathbb{R} \backslash\{0\}$ defined by

$$
\xi\left(t, k_{1}(t), k_{2}(t)\right)=\frac{t\left|\cos k_{1}(t)\right|}{\left(1+\left|\cos k_{1}(t)\right|\right) 10,000}+0.0001 t \int_{0}^{1} k_{2}(s) \mathrm{d} s+0.0009
$$

with $\xi^{*}=\sup _{t \in[0,1]}|\xi(t, 0,0)|=\frac{1}{20,000}+0.0009=0.00095$. On the other hand, it is clear that the function $\xi$ is Lipschitzian. Indeed, for each $k_{1}, k_{2} \in \mathbb{R}$, we have

$$
\begin{aligned}
& \left|\xi\left(t, k_{1}(t), \int_{0}^{1} k_{1}(s) \mathrm{d} s\right)-\xi\left(t, k_{2}(t), \int_{0}^{1} k_{2}(s) \mathrm{d} s\right)\right| \\
& \quad \leq \frac{t}{10,000}\left[\left|k_{1}(t)-k_{2}(t)\right|+\left|k_{1}(t)-k_{2}(t)\right|\right] \\
& \quad=\frac{2 t}{10,000}\left|k_{1}(t)-k_{2}(t)\right| .
\end{aligned}
$$

If we set $\theta(t)=\frac{t}{10,000}$, then $\theta^{*}=\sup _{t \in[0,1]}|\theta(t)|=0.0001$. In this position, we define the set-valued map $\mathcal{S}:[0,1] \times \mathbb{R}^{2+1} \rightarrow \mathcal{P}(\mathbb{R})$ by

$$
\begin{aligned}
\mathcal{S} & \left(t, k(t), \phi_{1}(k(t)), \phi_{2}(k(t))\right) \\
\quad & =\left[-2,(t+1) \cos k(t)+2 \sin \left(\mathcal{I}_{0}^{0.03} k(t)\right)+\frac{7}{10} \sin ^{2}\left(\mathcal{I}_{0}^{0.05} k(t)\right)+\frac{8}{10}\right] .
\end{aligned}
$$

Since

$$
|\zeta| \leq \max \left[-2,(t+1) \cos k(t)+2 \sin \left(\mathcal{I}_{0}^{0.03} k(t)\right)+\frac{7}{10} \sin ^{2}\left(\mathcal{I}_{0}^{0.05} k(t)\right)+\frac{8}{10}\right] \leq t+\frac{9}{2}
$$


for all $\zeta \in \mathcal{S}\left(t, k(t), \phi_{1}(k(t)), \phi_{2}(k(t))\right)$, we obtain

$$
\begin{aligned}
\left\|\mathcal{S}\left(t, k(t), \phi_{1}(k(t)), \phi_{2}(k(t))\right)\right\| & =\sup \left\{|\vartheta|: \vartheta \in \mathcal{S}\left(t, k(t), \phi_{1}(k(t)), \phi_{2}(k(t))\right)\right\} \\
& \leq t+4.5
\end{aligned}
$$

Put $q(t)=t+4.5$ for all $t \in[0,1]$. Then $\|q\|_{\mathcal{L}^{1}}=\int_{0}^{1}|q(s)| \mathrm{d} s=\int_{0}^{1}(s+4.5) \mathrm{d} s=5$ and $M \simeq$ 42.2585. Now, we choose $\tilde{\rho}>0$ so that

$$
\tilde{\rho}>\frac{\xi^{*} M\|q\|_{\mathcal{L}^{1}}}{1-2 \theta^{*} M\|q\|_{\mathcal{L}^{1}}}=\frac{0.00095 \times 42.2585 \times 5}{1-2(0.0001 \times 42.2585 \times 5)} \simeq 0.209574
$$

Thus $\tilde{\rho}>0.209574$. Then $4 \theta^{*} M\|q\|_{\mathcal{L}^{1}} \simeq 0.0845<1$. Now, by using Theorem 5 , the hybrid multi-term inclusion problem (10)-(11) has a solution.

\section{Conclusion}

It is known that most natural phenomena are modeled by different types of fractional differential equations and inclusions. This diversity in investigating complicate fractional differential equations and inclusions increases our ability for exact modelings of more phenomena. This is useful in designing modern software which helps us to allow for more cost-free testing and less material consumption. In this work, we study the existence of solutions for a fractional hybrid multi-term integro-differential inclusion problem with four-point sum and integral boundary value conditions. By using Dhage's fixed point results, we prove our main existence result. Finally, we give an example to illustrate our main result.

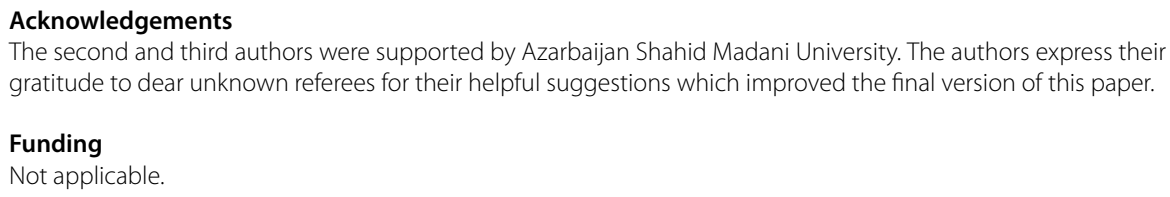

\section{Author details}

${ }^{1}$ Department of Mathematics, Cankaya University, Ankara, Turkey. ${ }^{2}$ Department of Mathematics, Azarbaijan Shahid Madani University, Tabriz, Iran. ${ }^{3}$ Department of Medical Research, China Medical University Hospital, China Medical University, Taichung, Taiwan. ${ }^{4}$ Institute of Space Sciences, Magurele, Bucharest, Romania.

\section{Publisher's Note}

Springer Nature remains neutral with regard to jurisdictional claims in published maps and institutional affiliations. 


\section{References}

1. Baleanu, D., Mohammadi, H., Rezapour, Sh.: On a nonlinear fractional differential equation on partially ordered metric spaces. Adv. Differ. Equ. 2013, 83 (2013). https://doi.org/10.1186/1687-1847-2013-83

2. Baleanu, D., Rezapour, Sh., Mohammadi, H.: Some existence results on nonlinear fractional differential equations. Philos. Trans. R. Soc. Lond. A 371, 20120144 (2013). https://doi.org/10.1098/rsta.2012.0144

3. Baleanu, D., Mohammadi, H., Rezapour, Sh.: The existence of solutions for a nonlinear mixed problem of singular fractional differential equations. Adv. Differ. Equ. 2013, 359 (2013). https://doi.org/10.1186/1687-1847-2013-359

4. Baleanu, D., Rezapour, Sh., Etemad, S., Alsaedi, A.: On a time-fractional integro-differential equation via three-point boundary value conditions. Math. Probl. Eng. 2015, Article ID 785738, 12 pages (2015)

5. Hedayati, V., Samei, M.E.: Positive solutions of fractional differential equation with two pieces in chain interval and simultaneous Dirichlet boundary conditions. Bound. Value Probl. 2019, 141 (2019) https://doi.org/10.1186/s13661-019-1251-8

6. Tunc, C., Tunc, O.: New qualitative criteria for solutions of Volterra integro-differential equations. Arab J. Basic Appl. Sci. 25(3), 158-165 (2018). https://doi.org/10.1080/25765299.2018.1509554

7. Talaee, M., Shabibi, M., Gilani, A., Rezapour, Sh.: On the existence of solutions for a pointwise defined multi-singular integro-differential equation with integral boundary condition. Adv. Differ. Equ. 2020, 41 (2020). https://doi.org/10.1186/s13662-020-2517-2

8. Baleanu, D., Hedayati, V., Rezapour, Sh., Al Qurashi, M.M.: On two fractional differential inclusions. SpringerPlus 5(1), 882 (2016). https://doi.org/10.1186/s40064-016-2564-z

9. Baleanu, D., Rezapour, Sh., Saberpour, Z.: On fractional integro-differential inclusions via the extended fractional Caputo-Fabrizio derivation. Bound. Value Probl. 2019, 79 (2019). https://doi.org/10.1186/s13661-019-1194-0

10. Ahmad, B., Etemad, S., Ettefagh, M., Rezapour, Sh.: On the existence of solutions for fractional q-difference inclusions with q-antiperiodic boundary conditions. Bull. Math. Soc. Sci. Math. Roum. 59(107)(2), 119-134 (2016). https://doi.org/10.1016/0003-4916(63)90068-X

11. Etemad, S., Ntouyas, S.K., Ahmad, B.: Existence theory for a fractional $q$-integro-difference equation with $q$-integral boundary conditions of different orders. Mathematics 71, 659 (2019)

12. Samei, M.E., Khalilzadeh Ranjbar, G., Hedayati, V.: Existence of solutions for equations and inclusions of multi-term fractional q-integro-differential with non-separated and initial boundary conditions. J. Inequal. Appl. 2019, 273 (2019). https://doi.org/10.1186/s13660-019-2224-2

13. Samei, M.E., Khalilzadeh Ranjbar, G., Hedayati, V.: Existence of solutions for equations and inclusions of multi-term fractional q-integro-differential with non-separated and initial boundary conditions. Kragujev. J. Math. 45(4), 543-570 (2021)

14. Kojabad, E.A., Rezapour, Sh.: Approximate solutions of a sum-type fractional integro-differential equation by using Chebyshev and Legendre polynomials. Adv. Differ. Equ. 2017, 351 (2017). https://doi.org/10.1186/s13662-017-1404-y

15. Aydogan, M.S., Baleanu, D., Mousalou, A., Rezapour, Sh.: On high order fractional integro-differential equations including the Caputo-Fabrizio derivative. Bound. Value Probl. 2018, 90 (2018). https://doi.org/10.1186/s13661-018-1008-9

16. Aydogan, S.M., Baleanu, D., Mousalou, A., Rezapour, Sh.: On approximate solutions for two higher-order Caputo-Fabrizio fractional integro-differential equations. Adv. Differ. Equ. 2017, 221 (2017) https://doi.org/10.1186/s13662-017-1258-3

17. Baleanu, D., Mousalou, A., Rezapour, Sh.: A new method for investigating approximate solutions of some fractional integro-differential equations involving the Caputo-Fabrizio derivative. Adv. Differ. Equ. 2017, 51 (2017). https://doi.org/10.1186/s13662-017-1088-3

18. Baleanu, D., Mousalou, A., Rezapour, Sh.: The extended fractional Caputo-Fabrizio derivative of order $0 \leq \sigma<1$ on $C_{\mathbb{R}}[0,1]$ and the existence of solutions for two higher-order series-type differential equations. Adv. Differ. Equ. 2018, 255 (2018). https://doi.org/10.1186/s13662-018-1696-6

19. Baleanu, D., Mousalou, A., Rezapour, Sh.: On the existence of solutions for some infinite coefficient-symmetric Caputo-Fabrizio fractional integro-differential equations. Bound. Value Probl. 2017, 145 (2017). https://doi.org/10.1186/s13661-017-0867-9

20. De La Sen, M., Hedayati, V., Gholizade Atani, Y., Rezapour, Sh.: The existence and numerical solution for a k-dimensional system of multi-term fractional integro-differential equations. Nonlinear Anal., Model. Control 22(2), 188-209 (2017)

21. Baleanu, D., Etemad, S., Pourrazi, S., Rezapour, Sh.: On the new fractional hybrid boundary value problems with three-point integral hybrid conditions. Adv. Differ. Equ. 2019, 473 (2019). https://doi.org/10.1186/s13662-019-2407-7

22. Baleanu, D., Khan, H., Jafari, H., Khan, R.A., Alipour, M.: On existence results for solutions of a coupled system of hybrid boundary value problems with hybrid conditions. Adv. Differ. Equ. 2015, 318 (2015). https://doi.org/10.1186/s13662-015-0651-z

23. Etemad, S., Rezapour, Sh., Samei, M.E.: On fractional hybrid and non-hybrid multi-term integro-differential inclusions with three-point integral hybrid boundary conditions. Adv. Differ. Equ. 2020, 161 (2020) https://doi.org/10.1186/s13662-020-02627-8

24. Khan, H., Tunc, C., Chen, W., Khan, A.: Existence theorems and Hyers-Ulam stability for a class of hybrid fractiona differential equations with p-Laplacian operator. J. Appl. Anal. Comput. 8(4), 1211-1226 (2018) https://doi.org/10.11948/2018.1211

25. Samei, M.E., Hedayati, V., Rezapour, Sh.: Existence results for a fraction hybrid differential inclusion with Caputo-Hadamard type fractional derivative. Adv. Differ. Equ. 2019, 163 (2019). https://doi.org/10.1186/s13662-019-2090-8

26. Samei, M.E., Khalilzadeh Ranjbar, G.: Some theorems of existence of solutions for fractional hybrid $q$-difference inclusion. J. Adv. Math. Stud. 12(1), 63-76 (2019)

27. Sun, S., Zhao, Y., Han, Z., Li, Y.: The existence of solutions for boundary value problem of fractional hybrid differential equations. Commun. Nonlinear Sci. Numer. Simul. 17, 4961-4967 (2012)

28. Tunc, C., Tunc, O.: A note on the qualitative analysis of Volterra integro-differential equations. J. Taibah Univ. Sci. 13(1), 490-496 (2019). https://doi.org/10.1080/16583655.2019.1596629 
29. Alizadeh, Sh., Baleanu, D., Rezapour, Sh.: Analyzing transient response of the parallel RCL circuit by using the Caputo-Fabrizio fractional derivative. Adv. Differ. Equ. 2020, 55 (2020). https://doi.org/10.1186/s13662-020-2527-0

30. Baleanu, D., Etemad, S., Rezapour, Sh.: A hybrid Caputo fractional modeling for thermostat with hybrid boundary value conditions. Bound. Value Probl. 2020, 64 (2020). https://doi.org/10.1186/s13661-020-01361-0

31. Baleanu, D., Ghafarnezhad, K., Rezapour, Sh., Shabibi, M.: On the existence of solutions of a three steps crisis integro-differential equation. Adv. Differ. Equ. 2018, 135 (2018). https://doi.org/10.1186/s13662-018-1583-1

32. Baleanu, D., Ghafarnezhad, K., Rezapour, Sh.: On a three steps crisis integro-differential equation. Adv. Differ. Equ. 2019, 153 (2019). https://doi.org/10.1186/s13662-019-2088-2

33. Baleanu, D., Jajarmi, A., Mohammadi, H., Rezapour, Sh.: A new study on the mathematical modeling of human liver with Caputo-Fabrizio fractional derivative. Chaos Solitons Fractals 134, 109705 (2020). https://doi.org/10.1016/j.chaos.2020.109705

34. Baleanu, D., Mohammadi, H., Rezapour, Sh.: Analysis of the model of HIV-1 infection of CD4+ T-cell with a new approach of fractional derivative. Adv. Differ. Equ. 2020, 71 (2020). https://doi.org/10.1186/s13662-020-02544-w

35. Dhage, B.C., Lakshmikantham, V.: Basic results on hybrid differential equation. Nonlinear Anal. Hybrid Syst. 4, 414-424 (2010)

36. Zhao, Y., Sun, S., Han, Z., Li, Q.: Theory of fractional hybrid differential equations. Comput. Math. Appl. 62, 1312-1324 (2011)

37. Hilal, K., Kajouni, A.: Boundary value problems for hybrid differential equations with fractional order. Adv. Differ. Equ. 2015, 183 (2015). https://doi.org/10.1186/s13662-015-0530-7

38. Ahmad, B., Ntouyas, S.K., Tariboon, J.: On hybrid Caputo fractional integro-differential inclusions with nonlocal conditions. J. Nonlinear Sci. Appl. 9(5), 4235-4246 (2016). https://doi.org/10.1063/1.5096159

39. Derbazi, C.H., Hammouche, H., Benchohra, M., Zhou, Y.: Fractional hybrid differential equations with three-point boundary hybrid conditions. Adv. Differ. Equ. 2019, 125 (2019)

40. Podlubny, l.: Fractional Differential Equations. Academic Press, San Diego (1999)

41. Samko, S.G., Kilbas, A.A., Marichev, O.I. Fractional Integrals and Derivatives: Theory and Applications. Gordon \& Breach, Philadelphia (1993)

42. Miller, K.S., Ross, B.: An Introduction to Fractional Calculus and Fractional Differential Equations. Wiley, New York (1993)

43. Deimling, K.: Multi-Valued Differential Equations. de Gruyter, Berlin (1992)

44. Aubin, J., Ceuina, A.: Differential Inclusions: Set-Valued Maps and Viability Theory. Springer, New York (1984)

45. Lasota, A., Opial, Z:: An application of the Kakutani-Ky Fan theorem in the theory of ordinary differential equations. Bull. Acad. Pol. Sci., Sér. Sci. Math. Astron. Phys. 13, 781-786 (1965)

46. Dhage, B.C.: Existence results for neutral functional differential inclusions in Banach algebras. Nonlinear Anal. 64 1290-1306 (2006)

\section{Submit your manuscript to a SpringerOpen ${ }^{\circ}$ journal and benefit from:}

- Convenient online submission

- Rigorous peer review

- Open access: articles freely available online

- High visibility within the field

- Retaining the copyright to your article

Submit your next manuscript at $\boldsymbol{\nabla}$ springeropen.com 